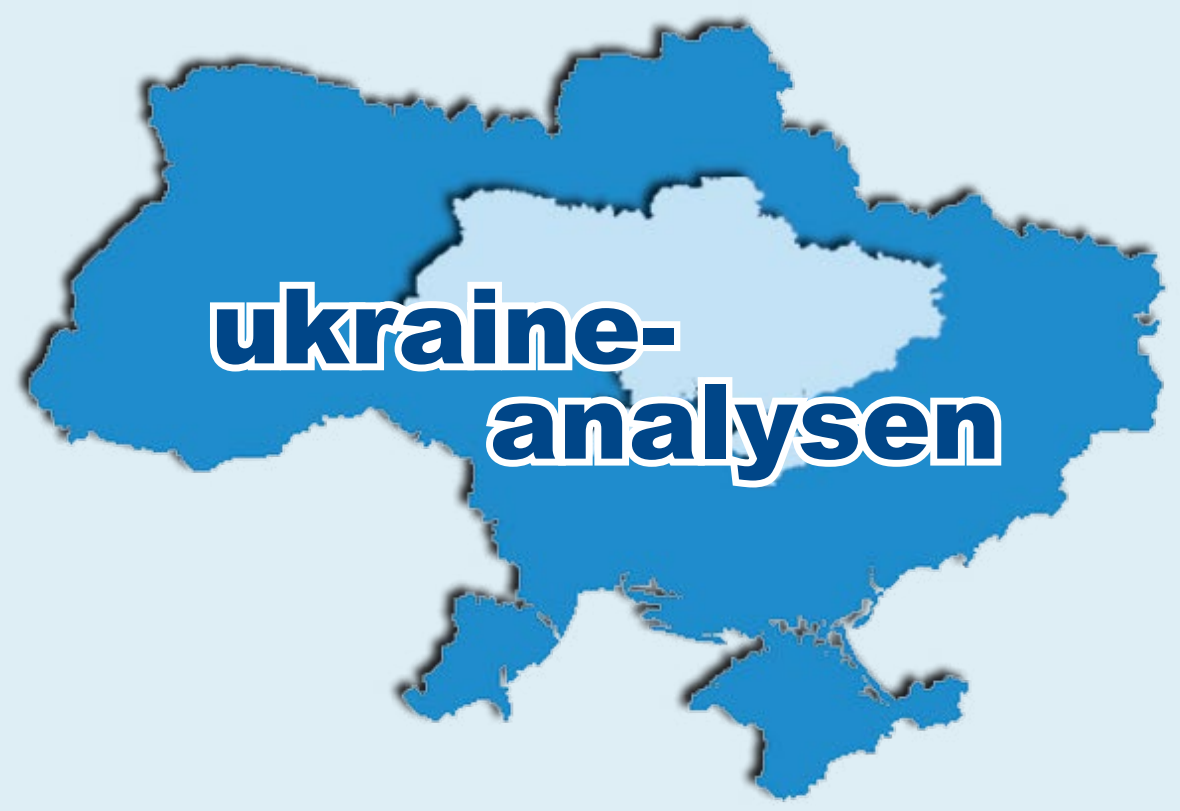

www.laender-analysen.de/ukraine

\title{
DIE KRIM
}

\section{DIE MINDERHEITENFRAGE}

ANALYSE

Russische Wirtschaftsförderung für die Krim - eine Zwischenbilanz

Von Julia Kusznir, Bremen

ANALYSE

»Es geht ums Geschäft«: Die Krim-Blockade und die Realität der

Wirtschaftsbeziehungen mit der Krim nach eineinhalb Jahren Annexion

Von Katerina Bosko, Bremen

TABELLEN UND GRAFIKEN ZUM TEXT

Ausgewählte Daten zu den Handelsbeziehungen der Ukraine mit der Krim

STATISTIK

Demographische Situation auf der Krim: aktuell und im Rückblick

DOKUMENTATION

Die Lage der Menschenrechte auf der Krim

Die Krim-Blockade in den Berichten der OSZE-Beobachtermission in der Ukraine (Auszüge)

ANALYSE

Die Rumänen und Ungarn der Ukraine - stille Minderheiten?

Von Oleg Friesen, München

CHRONIK

12. - 25. Oktober 2015 


\title{
Russische Wirtschaftsförderung für die Krim - eine Zwischenbilanz
}

\author{
Von Julia Kusznir, Bremen
}

\section{Zusammenfassung}

Nach der Annexion der Krim im März 2014 hat Russland große Investitionen in die Infrastruktur und eine wirtschaftliche Förderung der Region angekündigt. Bisher sind Erfolge dieser Maßnahmen jedoch nicht erkennbar. Die Probleme spitzen sich vielmehr dramatisch zu. Der Beitrag nimmt eine Bestandsaufnahme der wichtigsten Projekte vor.

\section{Einleitung}

Nach der Annexion der Republik Krim und der Stadt Sewastopol im März 2014 hat Russland ein umfangreiches wirtschaftliches Förderprogramm für die Halbinsel ins Leben gerufen, das die beiden neuen Föderationssubjekte (d. h. Regionen) der Russischen Föderation in den kommenden Jahren zu einer der wirtschaftlich dynamischsten Regionen Russlands entwickeln sollte.

Für die Ausarbeitung der Maßnahmen und die Kontrolle ihrer Umsetzung wurde eigens ein Ministerium für Angelegenheiten der Krim geschaffen. Bis zum Jahr 2020 sollen aus dem russischen Staatshaushalt mehr als 20 Milliarden US-Dollar als Wirtschaftshilfe auf die Halbinsel fließen. Der wesentliche Teil der Investitionen in die Infrastrukturprojekte soll aber von russischen Unternehmen getragen werden. Um das Investitionsklima auf der Krim zu verbessern, sind u. a. Vorzugskredite und Steuervergünstigungen für russische Unternehmen vorgesehen.

\section{Umsetzungsprobleme}

Eines der Hauptziele der russischen Projekte auf der Krim war es, die Halbinsel in den Bereichen Energie- und Wasserversorgung sowie Transport von der Ukraine unabhängig zu machen. Anderthalb Jahre nach der Annexion der Krim konstatieren russische Regierungsvertreter, dass die Wirtschaftslage der Krim weiterhin schwierig ist und der Zustand der Infrastruktur schlecht. Die Gründe dafür seien die Transportblockade durch die Ukraine, die Blockade der Wasserversorgung sowie die Energieabhängigkeit der Halbinsel vom ukrainischen Festland.

Aufgrund zurückgehender Staatseinnahmen in Folge des Einbruchs des Weltmarktpreises für Erdöl hat die russische Regierung außerdem Probleme, die für die Krim vorgesehenen Finanzmittel aufzutreiben. Zur Effizienzsteigerung wurde das Ministerium für Angelegenheiten der Krim bereits im Juli 2015 wieder aufgelöst, seine Aufgaben wurden an das Wirtschaftsministerium übergeben. Dieses hat Ende Oktober mitgeteilt, dass die staatliche Finanzierung einiger für dieses Jahr geplanter Projekte in das nächste Jahr verschoben wird, sprich: Für die Krim-Projekte fehlt derzeit das Geld.
Gleichzeitig beklagt die russische Behörde Misswirtschaft und Inkompetenz bei der regionalen Verwaltung der Krim. Dies führt immer wieder zu Skandalen wegen Unterschlagungen und Korruption. Seit der Annexion durch Russland wurden ca. 700 Korruptionsfälle auf der Krim registriert, von denen 60 mit dem Missbrauch von Geldern aus dem russischen Staatshaushalt verbunden war. Um Korruption zu bekämpfen und die Effizienz der regionalen Verwaltung zu erhöhen, beschloss die russische Regierung, die staatlichen Behörden auf der Krim durch Beamte aus der russischen Staatsverwaltung zu verstärken. Es wird darüber spekuliert, ob das russische Innenministerium und das Verteidigungsministerium diese Aufgabe übernehmen. Diese zwei Ministerien werden von den so genannten "Silowiki«, Vertretern der staatlichen Sicherheitsstrukturen, kontrolliert.

\section{Wasserversorgung}

Die Halbinsel Krim erhielt bis zu 85 \% ihrer Wasserversorgung, insgesamt mehr als eine Milliarde Kubikmeter, über den Nord-Krim-Kanal aus dem ukrainischen Teil des Flusses Dnjepr. Im Mai 2014 hat die Ukraine die Wasserlieferungen auf die Krim eingestellt, mit der Begründung, dass keine neuen vertraglichen Vereinbarungen zwischen den ukrainischen Behörden und der Regierung der Krim erreicht werden konnten.

Die Wasserknappheit war vor allem für die Landwirtschaft auf der Krim spürbar. Aus diesem Grund wurde z. B. der Anbau von Reis eingestellt und der von Mais und Soja wesentlich reduziert. Große Probleme gab es auch bei der Trinkwasserversorgung der großen Industriestädte der Halbinsel etwa Kertsch und Feodosia. Nach offiziellen Angaben wurde der Wasserverbrauch der Krim in den vergangenen zwei Jahren auf ein Fünftel reduziert.

Um die Wasserversorgung für die Bewohner der Krim zu gewährleisten, wurden artesianische Brunnenwasserquellen und Stauseen aktiviert und zusätzliche Wasserleitungen gebaut. Die Behörden gehen davon aus, dass die vorhandenen Wasservorräte bis zum Frühjahr 2016 ausreichen. Längerfristig gibt es hingegen bisher keine Alternativen zur Wasserversorgung aus der Ukraine und damit auch keine klare Lösung für die Wasserversorgung der Krim. 


\section{Energieversorgung}

Genau wie beim Wasser kamen auch beim Strom mehr als $80 \%$ des Verbrauchs der Halbinsel Krim aus der Ukraine, das entspricht über sechs Milliarden Kilowattstunden. Der restliche Bedarf wird aus Eigenproduktion gedeckt.

Um die Energieunabhängigkeit der Krim zu gewährleisten, hat Russland eine Reihe von Infrastrukturprojekten geplant. Sie umfassen unter anderem den Bau von zwei Wärmekraftwerken mit einer Gesamtkapazität von 700 Megawatt in Simferopol und Sewastopol. Die Kraftwerke sollen mit russischem Gas beliefert werden. Dafür wird die Gaspipeline Kuban-Krim gebaut, die mit einer Jahreskapazität von vier Milliarden Kubikmetern von der russischen Region Krasnodar durch die Straße von Kertsch laufen wird und bis März 2017 fertiggestellt werden soll.

Darüber hinaus sehen die russischen Infrastrukturprogramme für die Halbinsel die Modernisierung des Stromnetzes vor. Zusätzlich ist eine Verbindung mit dem russischen Stromnetz über die Verlegung eines Stromkabels durch die Straße von Kertsch geplant. Auch der Bau von Anlagen für Stromerzeugung aus erneuerbaren Energiequellen ist vorgesehen.

Nach Einschätzung der russischen Behörden wird es noch fünf bis sechs Jahre dauern, bis die Krim von Stromlieferungen aus der Ukraine unabhängig ist. Die westlichen Sanktionen verhindern aber die Auslieferung der für die Kraftwerke notwendigen Turbinen. Die in Russland hergestellten Turbinen sind qualitativ deutlich schlechter und stellen keine gute Lösung dar. Darüber hinaus führen steigende Kosten und schrumpfende Finanzierungsmöglichkeiten zur Verschiebung einiger Projekte.

Deshalb hat sich Russland mit der Ukraine auf die Fortsetzung der Stromversorgung der Krim geeinigt. Seit Juli 2014 bezieht die Krim ukrainischen Strom nicht mehr zum subventionierten Inlandspreis, sondern bezahlt den vollen Exportpreis. Seit September 2014 hat die Ukraine ihre Stromlieferungen auf die Krim aber kontinuierlich reduziert. Ursache hierfür ist nach Angaben des ukrainischen Netzwerkbetreibers das Fehlen von Kohle für die Wärmekraftwerke des Landes aufgrund der Zerstörung von Verkehrswegen und Bergwerken durch die pro-russischen Separatisten in der Ostukraine (s. Ukraine-Analysen Nr. 157).

Die Stromversorgung der Krim ist in der Ukraine politisch zunehmend umstritten. Im Rahmen einer Blockade von Warenlieferungen über Zufahrtsstraßen zur Krim fordern sowohl Vertreter der Krimtataren als auch nationalistische politische Organisationen wie der Rechte Sektor eine Energieblockade der Halbinsel. Zusätzlich haben - entsprechend einer neu geschaf- fenen Möglichkeit - 25.000 Personen eine elektronische Petition zur Einstellung der Stromversorgung der Krim unterzeichnet. Die Petition hat keinen verbindlichen Charakter, verpflichtet den Präsidenten aber zu einer Stellungnahme. Die ukrainische Präsidialverwaltung hat erst einmal erklärt, dass sie Verständnis für die Proteste hat, die Blockade aber vom ukrainischen Staat nicht unterstützt wird.

\section{Verkehrsanbindung und Tourismus}

Infolge der Annexion hat sich der Reise- und Güterverkehr auf die Krim wesentlich verändert. Ende 2014 hat die staatliche ukrainische Eisenbahn den Zugverkehr auf die Halbinsel aus Sicherheitsgründen dauerhaft eingestellt. Eine funktionierende Schienenanbindung an das russische Festland fehlt weiterhin.

Im Ergebnis hat der Luftverkehr auf die Krim deutlich zugenommen, seit 2013 hat er sich verdreifacht. Da die Annexion der Krim international nicht anerkannt wurde und der Luftraum über der Krim damit formal zur Ukraine gehört, können nur russische Fluggesellschaften die Krim anfliegen. Die Ukraine hat jedoch gegen die russischen Fluggesellschaften, die die Krim anfliegen, jeweils Strafen wegen der Verletzung ihres Luftraums verhängt. Ende 2014 beliefen sich die Strafen auf mehr als 18 Millionen US-Dollar. Im September 2015 reagierte die Ukraine mit einem (teilweisen oder vollständigen) Verbot von Transitflügen russischer Fluggesellschaften über ukrainisches Territorium. Das Verbot ist am 25. Oktober 2015 in Kraft getreten. Die Situation des Flugverkehrs auf die Krim wird damit noch komplizierter.

Die Fährverbindung über die Straße von Ketsch ist weiterhin die einzige größere Verkehrsverbindung zum russischen Festland. Die Passagierzahl der Fährverbindung belief sich 2014 auf mehr als 2,3 Millionen Menschen. Russland hofft aber, dass mit dem Bau der Auto- und Eisenbahnbrücke über die Meerenge von Kertsch die Probleme der direkten Verkehrsanbindung der Krim endgültig gelöst werden. Der Bau dieser Brücke ist bereits 2004 als gemeinsames russisch-ukrainisches Projekt geplant worden. Nach der Annexion der Krim begann Russland die technischen Arbeiten am Projekt, ohne die ukrainischen Partner einzubeziehen. Die ukrainische Regierung hat in Reaktion darauf ihren Ausstieg aus dem Projekt beschlossen.

Mit dem Bau der Brücke hat die russische Regierung das Unternehmen Strojgazmontazh beauftragt, das zum SGM-Konzern des Milliardärs und engen Freundes von Präsident Putin Arkadi Rotenberg gehört. Die Arbeiten zum Bau der Brücke wurden im Oktober 2015 aufgenommen und sollen Ende 2018 abgeschlossen werden. Mit geschätzten vier Milliarden US-Dollar Baukosten 
wird die Brücke von Experten als teuerste russische Baumaßnahme des 21. Jahrhunderts bezeichnet.

Die Annexion war auch ein schwerer Schlag für die Tourismusbranche auf der Krim. Während die Infrastrukturprobleme die Einreise von russischen Touristen auf die Krim wesentlich erschwert haben, sind die ukrainischen Touristen gleichzeitig weitgehend ausgeblieben. Die Zahl der ukrainischen Touristen auf der Krim sank von 4,5 Millionen im Sommer 2013 auf weniger als 0,2 Millionen 2015. Der Rückgang hat nicht nur politische Gründe, sondern hängt auch mit den durch die Angliederung an Russland gestiegenen Lebenshaltungskosten auf der Krim zusammen, die derzeit im Durchschnitt 60 bis 70 Prozent höher sind als in der Ukraine.

Im Juni 2015 hat die ukrainische Regierung neue Ein- und Ausreiseregeln für die Krim eingeführt. Während ukrainische Bürger die Krim mit einem Reisepass besuchen können, müssen Ausländer eine gesonderte Genehmigung der ukrainischen Migrationsbehörde einholen. Sie wird allerdings nur unter bestimmten Voraussetzungen wie z. B. für den Besuch von auf der Krim lebenden Verwandten erteilt. Reisen auf die Krim für touristische Zwecke werden Ausländern von Seiten der Ukraine nicht genehmigt.

\section{Verstaatlichung von Unternehmen auf der Krim}

Um die regionale Stabilität und Energiesicherheit zu gewährleisten, hat der Staatsrat der Republik Krim am 26. März 2014 eine Resolution »Über die Verstaatlichung von Unternehmen, Institutionen und Organisationen des Agrar- und Industrie-Komplexes in der Republik Krim» verabschiedet und auf deren Grundlage vom Frühjahr 2014 bis zum Frühjahr 2015 fast 500 Großunternehmen und Organisationen, die sich im Besitz des ukrainischen Staates oder ukrainischer Privateigentümer befanden, beschlagnahmt. So wurden zum Beispiel im März 2014 die regionalen Energieunternehmen Chernomorneftegaz und Ukrtransgaz verstaatlicht. Beide waren Tochterfirmen des staatlichen ukrainischen Energiekonzerns Naftogaz.

Im September 2014 genehmigte der Staatsrat der Krim außerdem die Verstaatlichung des gesamten Vermögens einiger ukrainischer Großunternehmer auf dem Territorium der Krim. So wurden etwa die Aktiva des ehemaligen Gouverneurs der Region Dnipropetrowsk, des Oligarchen Ihor Kolomojskyj, beschlagnahmt. Kolomojskyj selbst schätzte seine Vermögensverluste auf zwei Milliarden US-Dollar.

Als nächstes wurde das Vermögen des Energiekonzerns Krymgaz, das dem ukrainischen Oligarchen Dmytro Firtasch gehörte, vom russischen Staat übernommen. Das Unternehmen betreibt unter anderem eine 9.500
Kilometer lange Gaspipeline, durch die sie die meistbesiedelten Städte und Bezirke auf der Krim beliefert.

In Januar 2015 folgte die Verstaatlichung des Energieunternehmens DTEK Krymenergo, das dem ukrainischen Oligarchen Rinat Achmetow gehörte. Das Unternehmen verfügte über ein Monopol bei der Stromversorgung der Krim. Einen Monat später, im Februar 2015, beschlagnahmten die staatlichen Behörden eine weitere Firma von Achmetow - Ukrtelecom, einen der führenden Festnetz- und Mobilfunkbetreiber auf der Halbinsel.

Im selben Monat wurde auch eine der größten Schiffswerften der Krim, OAO Sevmorzavod, verstaatlicht, die sich im Besitz des ukrainischen Präsidenten Poroschenko befand.

Als nächstes planen die Behörden der Krim eine Reihe von Privatisierungsauktionen, um die verstaatlichten Unternehmen zur Sanierung des regionalen Staatshaushaltes zu verkaufen. In Reaktion hierauf hat die ukrainische Regierung angekündigt, Russland für jeden einzelnen Fall von Eigentumsverlust auf der Krim vor internationalen Gerichten zu verklagen. Die Höhe der entsprechenden Schadensersatzforderungen wird von ukrainischer Seite derzeit auf 45 Milliarden US-Dollar geschätzt.

\section{Fazit}

Nach der Annexion der Krim hat die russische Regierung intensiv versucht, die Probleme der Wasser- und Energieabhängigkeit von der Ukraine sowie die Probleme der Verkehrsanbindung zu lösen. Da dies kurzfristig aber nicht möglich ist, leidet die Krim unter der teilweisen Blockade durch die Ukraine sowie unter den westlichen Sanktionen, die sowohl den internationalen Schiffs- und Flugverkehr als auch die Geschäftsbeziehungen von Unternehmen auf der Krim drastisch einschränken.

Da die Ukraine selber von russischen Erdgaslieferungen abhängig ist, hat sie mit der Energieversorgung der Krim ein Faustpfand für die Verhandlungen mit Russland. Damit wird weiterhin eine gewisse gegenseitige Abhängigkeit zwischen Russland und der Ukraine bestehen. Dies kann von beiden Seiten auch als politisches Instrument im bestehenden Konflikt genutzt werden.

Der Konflikt zwischen der Ukraine und Russland scheint derzeit nicht lösbar und damit ist auch eine Aufhebung der westlichen Sanktionen nicht absehbar. Die Probleme des völkerrechtlichen Status der Krim, die westlichen Sanktionen und die russische Wirtschaftskrise werden die notwendigen Investitionen in die regionalen Infrastrukturprojekte stark erschweren, so dass sich die Wirtschaftslage auf der Krim verschlechtern dürfte. Deshalb ist mit einem Anstieg der Arbeitslosig- 
keit zu rechnen, womit auch das Potential für soziale Proteste steigt.

Zusammenfassend lässt sich festhalten, dass die ambitionierten Pläne Russlands unter den gegebenen
Umständen nicht realisiert werden können, so dass die Krim auch zukünftig eine große finanzielle Belastung für Russland sein wird.

\section{Über die Autorin}

Dr. Julia Kusznir war Postdoctoral Fellow an der Jacobs University in Bremen. Schwerpunkte ihrer Forschung sind Föderalismus und Regionalisierungsprozesse in Russland, Stabilisierung und Konsolidierung autoritärer Regime, die Interaktionen der Akteure in Wirtschaft und Politik sowie Formen und Auswirkungen informeller Institutionen und Praktiken auf die politischen Prozesse in GUS-Staaten.

\section{Lesetipps}

- Berman, Ilan (2015) Paradise Lost in Crimea. How Russia Is Paying for the Annexation, Foreign Affairs, 08.09.2015, $<$ https://www.foreignaffairs.com/articles/ukraine/2015-09-08/paradise-lost-crimea>

- Paul, Amanda (2015) Crimea one year after Russian annexation, Policy Paper, European Policy Centre,24.03.2015, <http://www.epc.eu/documents/uploads/pub_5432_crimea_one_year_after_russian_annexation.pdf>

- Thomas de Waal (2015) The New Siege of Crimea, The National Interest, 09.07.2015, <http://nationalinterest.org/ feature/the-new-siege-crimea-13291>

\section{ANALYSE}

\section{»Es geht ums Geschäft«: Die Krim-Blockade und die Realität der Wirtschaftsbeziehungen mit der Krim nach eineinhalb Jahren Annexion}

Von Katerina Bosko, Bremen

\section{Zusammenfassung}

Dank der Krim-Blockade durch Krimtataren und Aktivisten der rechten Szene ist die Krim-Frage seit September wieder auf der Tagesordnung. Die Blockade hat die Aufmerksamkeit aber nicht nur auf die Menschenrechtsfrage, sondern auch auf die Handelsbeziehungen der Ukraine mit der Krim gelenkt. In diesem Artikel wird analysiert, wie die ukrainischen Behörden mit der neuen Realität auf der Krim umgehen. Ein besonderer Fokus liegt dabei auf der Steuerung der Abhängigkeiten der Krim vom ukrainischen Festland.

\section{Die Krim-Blockade}

Am 20. September 2015 haben die Krimtataren zusammen mit anderen Aktivisten die Durchfahrt in die Krim an allen Kontrollpunkten in der Region Cherson blockiert. Die seitdem geltende Blockade betrifft nur Lastkraftwagen, die Waren - hauptsächlich Lebensmittel transportieren. Bürger und Personenkraftwagen können die Grenze zur Halbinsel auch weiterhin ungehindert überqueren. Eine Woche später haben Aktivisten auch eine Bahnlinie blockiert, die zu zwei Fabrikanlagen des Oligarchen Dmytro Firtasch führt. Anfang Oktober kam zur Lebensmittelblockade die Sabotage von Stromlieferungen hinzu. So haben Unbekannte am 6. Oktober eine von insgesamt vier in die Krim verlegten Stromleitungen beschädigt. Die Aktion hatte für die Stromversorgung der Krim aber kaum Folgen, denn eine andere
Stromleitung wurde sofort außerplanmäßig in Betrieb genommen. Schon am 13. Oktober hatte der ukrainische Stromexporteur Ukrinterenergo die beschädigte Stromleitung repariert.

Die jetzige Krim-Blockade ist nicht die erste. Am 4. Juni 2015 hat die ukrainische Regierung eine Ein- und Ausreiseregelung für das vorübergehend besetzte Gebiet der Autonomen Republik Krim genehmigt. Nach der neuen Verordnung ist die Durchfahrt nur an bestimmten Kontrollpunkten möglich, die rund um die Uhr arbeiten. Mit den neuen Kontrollen wurden nicht nur neue Möglichkeiten zur Korruption geschaffen, sondern auch eine schnelle Absperrung der Verkehrswege ermöglicht. Ein erster »Test « fand Ende Juni 2015 statt, als der LKW-Verkehr an zwei von insgesamt drei Punkten für zwei Tage gestoppt wurde. Damals erklärte die ukrai- 
nische Seite den Zwischenfall mit einer Operation der Sicherheitskräfte gegen Schmuggel.

Die Initiatoren der Herbst-Blockade sind nicht nur die Krimtataren. Eine Reihe von anderen Organisationen wie etwa der Rechte Sektor, der Automaidan und der Maidan Selbstverteidigung, aber auch Vertreter der Bataillone Aidar und Donbas helfen bei der Durchführung der Aktion (s. OSZE-Dokumentation auf S. 16). Die große Anzahl von Teilnehmern ist durch eine Interessenskoinzidenz verschiedener Akteursgruppen zu erklären, deren gemeinsamer Nenner die Rückkehr der Krim zur Ukraine ist. So ist eine der Hauptforderungen die Abschaffung der freien Wirtschaftszone Krim, durch die nach Meinung der Aktivisten das "Besatzungsregime " auf der Krim finanziert und Superprofite erwirtschaftet werden. Der Vorsitzende des Medschlis des krimtatarischen Volkes Refat Tschubarow hat zum Beispiel zur Schaffung von Einzelhandelsmärkten an der ukrainischen Grenze für Krim-Bewohner aufgerufen. Dadurch würden die Oligarchen zu Transparenz gezwungen, während die erwirtschafteten Gewinne in der Ukraine verbleiben würden.

Die Ziele der Krimtataren gehen aber weit über die Korruptionsvorwürfe hinaus. Noch am 8. September haben sie eine Anzahl von Forderungen an die Russische Föderation gestellt. Ihnen zufolge sollen die russischen Behörden die Verfolgung der Krimtataren und anderer Bürger der Ukraine auf der Krim stoppen, die ukrainischen politischen Gefangenen freilassen, die Hindernisse für die Arbeit krimtatarischer und ukrainischer Medien auf der Krim beseitigen und das Einreiseverbot für die Anführer der Krimtataren Mustafa Dschemiljew und Refat Tschubarow aufheben. Die Blockade wurde somit mit der Menschenrechtsfrage auf der Halbinsel verknüpft (siehe Dokumentation auf S. 14). Diese Motivation wird an einer Aussage Tschubarows in einem Interview deutlich - »sie [die Russen - KB] misshandeln uns und wir beliefern sie mit Waren«, das sei abnorm.

Die Anführer der Krimtataren sind sich ihrer Grenzen bewusst. Sie streben keine "Befreiung" der Krim durch Militäraktionen an, stattdessen wollen sie Druck auf den Kreml ausüben. Mit der Krim-Blockade hoffen sie, Russland zu Gegenmaßnahmen zu bewegen, was ein Schlag für Russlands Staatsbudget wäre. Schließlich rechnen die Anführer der Krimtataren damit, dass auf der Krim die Unzufriedenheit mit der neuen Regierung wächst. Dass das Unbehagen in offene Proteste umschlägt, glauben sie aber nicht. Die Angst vor Repressionen sei hoch, wenn die Zeit reif für eine Rückkehr der Krim sei, würde jedoch keiner ein Verbleiben der Krim in der Russischen Föderation verteidigen wollen, so die Logik.

Offiziell wurde die Krim-Blockade von den ukrainischen Behörden nicht unterstützt. Allerdings wäre eine solche Aktion ohne deren stillschweigende Einwilligung nicht möglich. Außerdem hat die Cherson-Miliz die Blockade nicht verhindert und später sogar bei Fahrzeugüberprüfungen geholfen (s. OSZE-Dokumentation auf S. 16). Für die Regierung in Kiew ist die Aktion in vielerlei Hinsicht vorteilhaft. Zum einen nutzt Präsident Petro Poroschenko die Frage der Krimtataren, um seine außenpolitische Ziele zu befördern. Am 29. September sprach er bei der 70. UN-Generalversammlung über die Menschenrechtslage auf der Krim. Die Proteste der Krimtataren sind also ein zusätzliches Argument gegenüber Russland, das den Vorwurf der Unterdrückung der indigenen Bevölkerung auf der Halbinsel für unbegründet hält. Zum anderen kann die Krim-Blockade als Instrument zur Gestaltung der Beziehungen zwischen der Regierung und den Teilen der Wirtschaft dienen, die Interesse am Handel mit der Krim haben.

Die Aufnahme der Freilassung von politischen Gefangenen, unter anderem auch der ukrainischen Kampfpilotin Nadeschda Sawtschenko, auf die Liste der Forderungen der Krimtataren deutet darauf hin, dass die Aktion mit Kiew koordiniert wurde. Schon im letzten Jahr hat Poroschenko eine Kooperation mit den krimtatarischen Führern etabliert. Im Oktober 2014 wurden Tschubarow und Dschemiljew über die Liste des Blocks Petro Poroschenko ins Parlament gewählt. Nach dem Beginn der Krim-Blockade ist diese Zusammenarbeit noch enger geworden. Am 26. September 2015 hat Poroschenko Dschemiljew zum Leiter des Nationalen Rats für Antikorruptionspolitik, ein Beratungsgremium des Präsidenten, ernannt. Außerdem haben die rechtsradikalen Organisationen Dschemiljew am 13. Oktober 2015 für die gute Zusammenarbeit mit dem nichtstaatlichen Orden "Volksheld der Ukraine« ausgezeichnet. Vor ihm haben den im Jahr 2015 eingeführten Orden zum Beispiel der Anführer des Rechten Sektors Dmytro Jarosch und einige Mitglieder der Bataillone Aidar und Donbas bekommen.

\section{Freie Wirtschaftszone Krim}

Seit September 2014 gilt in der Ukraine das Gesetz über die freie Wirtschaftszone Krim, demzufolge die Ukraine auf der Krim zehn Jahre lang keine Steuern oder Gebühren erhebt. Ende November 2014 hat auch Russland ein ähnliches Gesetz verabschiedet, das Anfang 2015 in Kraft tritt und eine Geltungsdauer von 25 Jahren hat. Seit dem 1. Januar 2015 ist die Halbinsel Krim somit eine neue Steueroase und trotz der Sanktionen ein attraktiver Standort für lukrative Geschäfte.

Das wahre Ausmaß der Geschäftsbeziehungen mit der Krim ist aber schwer zu beurteilen. Seit der Annexion gibt das ukrainische Statistikamt keine Daten für die Krim mehr heraus. Laut russischem Statistikamt 
beliefen sich die Warenimporte aus der Ukraine von Januar bis August 2015 auf 36,4 Millionen US-Dollar (s. Tabelle 2 auf S. 10). Höchstwahrscheinlich ist diese Schätzung aber bei weitem zu niedrig. Laut ukrainischen Medien, die sich auf Angaben des Zollamts der Ukraine beziehen, wurden im gleichen Zeitraum Waren im Wert von 610 Millionen US-Dollar in die Krim geliefert und seit der Schaffung der freien Wirtschaftszone Krim auf diese Weise über eine Milliarde US-Dollar verdient. Zum Vergleich: Russland bezuschusst den KrimHaushalt in diesem Jahr mit etwa 800 Millionen USDollar (50,9 Milliarden Rubel).

Aus der Ukraine werden hauptsächlich Lebensmittel importiert. Schon vor der Annexion war die Lebensmittelindustrie der Krim von Lieferungen vom ukrainischen Festland abhängig. Unabhängig vom rechtlichen Status der Krim wurde der Handel somit fortgesetzt. Verglichen mit den Vorjahren ist das Geschäft aber viel profitabler geworden. Nach der Eingliederung in die Russische Föderation wurden die Preise für Lebensmittel um 50 Prozent angehoben (s. Ukraine-Analysen Nr. 141). Die Preisunterschiede wurden durch die Beseitigung der Doppelbesteuerung aber nur verfestigt. Deshalb sind die ukrainischen Nahrungsprodukte auf den Krim-Märkten viel günstiger als einheimische oder russische Lebensmittel. Zum einen leidet die Agrarwirtschaft der Krim aufgrund der Einstellung der Wasserlieferungen über den Nord-Krim-Kanal durch die Ukraine unter Wasserknappheit. Zum anderen reichen die Lebensmittellieferungen aus Russland aufgrund der ungünstigen Verbindung der Halbinsel mit Russland nicht aus. Nach offiziellen Angaben kostete im September 2015 ein Kilogramm Kartoffeln im Durchschnitt etwa 80 Prozent, ein Kilogramm Zucker 55 Prozent und ein Liter Milch 46 Prozent mehr als auf dem ukrainischen Festland (s. Tabelle 1 auf S. 9). Ukrainischen Medien zufolge werden die preisgünstigen Lebensmittel aus der Ukraine auch weiter nach Russland transportiert.

Die Krim-Blockade hat die Lebensmitteleinfuhr aus der Ukraine deutlich reduziert, wenn nicht gestoppt. Wie lange die Blockade noch dauert, ist nicht klar. Inzwischen hat Russland aber nach neuen Transportwegen gesucht. Im Oktober 2015 gab die russische Bundesaufsichtsbehörde für Tier- und Pflanzengesundheit die Aufnahme von Lebensmittellieferungen aus der Türkei trotz der Sanktionen bekannt. Derzeit wird die Möglichkeit einer neuen Fährverbindung mit der Türkei diskutiert.

\section{Umstrittene Stromlieferungen}

Die Krim ist neben den Lebensmitteln auch von der Stromversorgung aus der Ukraine abhängig. Ende Dezember 2014 haben das ukrainische Energieunter- nehmen Ukrinterenergo und der russische Energiekonzern Inter RAO einen Vertrag über Stromlieferungen in die Krim unterzeichnet. Laut diesem Vertrag verpflichtet sich die Ukraine, 550 Millionen Kilowattstunden Strom pro Monat auf die Halbinsel Krim zu liefern. Es wurde ein Strompreis von 2,44 Rubel pro Kilowattstunde vereinbart.

Zum Vergleich: Im Jahr 2014 wurde der ukrainische Strom durch Rinat Achmetows DTEK Krymenergo zum Preis von 3,42 Rubel pro Kilowattstunde auf der Krim verkauft. Nach der Annexion der Krim hat der Oligarch die Stromversorgung der Krim wie folgt organisiert: Das ukrainisch registrierte DTEK Krymenergo kaufte Strom auf dem ukrainischen Markt an und verkaufte ihn dann an die russische Inter RAO, die ihn zu einem niedrigeren Preis dem russisch registrierten DTEK Krymenergo auf der Krim verkaufte. Der Preisunterschied wurde aus dem russischen Staatsbudget finanziert. Nach der Unterzeichnung des Stromliefervertrags mit Ukrinterenergo wurde Rinat Achmetow allerdings aus dem Geschäft geworfen, DTEK Krymenergo wurde Anfang 2015 von den russischen Behörden verstaatlicht.

In der Ukraine war der neue Stromliefervertrag mit Russland sofort umstritten. Ende Januar 2015 sickerte er an die Medien durch. Gleichzeitig wurde eine Anordnung des Regierungsministers Arseniy Jazenjuk an den Energieminister Volodymyr Demtschyschyn bekannt gemacht. Mit ihr forderte Jazenjuk Aufklärung darüber, warum die Ukraine den Stromliefervertrag mit den für sie ungünstigen Bedingungen unterzeichnet hat. So sei der vereinbarte Strompreis niedriger als der Preis des bisherigen Anbieters - DTEK Krymenergo. Außerdem habe die Ukraine die Krim als Teil Russlands vertraglich anerkannt, so Jazenjuk.

Trotz der Rücktrittsforderungen seitens der Radikalen Partei Ljaschkos und der Partei Swoboda wurde der amtierende Generaldirektor von Ukrinterenergo nicht entlassen. Kurz vor der Unterzeichnung des umstrittenen Stromliefervertrags wurde die Leitung von Ukrinterenergo innerhalb eines Monats dreimal ausgetauscht. Anscheinend verharrt die Regierung auch weiterhin auf dem Status Quo. Im Oktober 2015 endete der Wettbewerb um die Besetzung der Führungsposition bei Ukrinterenergo wieder erfolglos.

Mittlerweile ist klar, dass die Regierung den Stromliefervertrag nicht kündigen will, sondern an seiner Verlängerung interessiert ist. Denn nach Aussagen des ukrainischen Energieministers ist dieser Vertrag für die Ukraine äußerst profitabel. Der Stromliefervertrag in die Krim wurde damals an einen anderen Vertrag mit Inter RAO über Stromimporte aus Russland geknüpft. Aufgrund der Kämpfe im Donbas ging die 
Förderung der Kohle, die in der Ukraine zur Stromerzeugung genutzt wird, stark zurück (s. Ukraine-Analysen Nr. 157). Ende November 2014 führte eine Stromknappheit zu abnehmenden Stromlieferungen in die Krim. Das Problem wurde über zusätzliche Stromimporte aus Russland gelöst. Seit Beginn des Jahres hat die Ukraine etwa 2.072 Millionen Kilowattstunden Strom aus Russland importiert, was weit unter dem vereinbarten Exportvolumen für die Krim liegt (6.600 Millionen Kilowattstunden). Nach Einschätzungen der Autorin entspricht der Nettogewinn der Ukraine gemäß den Geschäftsbedingungen im ursprünglichen Stromliefervertrag mindestens 100 Millionen US-Dollar (s. Tabelle 3 auf S. 9). Seit die Ukraine die Strompreise für die Krim im Juli auf 3,42 Rubel pro Kilowattstunde und im September auf 3,95 Rubel pro Kilowattstunde erhöht hat, stieg der Nettogewinn um ca. 40 Millionen US-Dollar. Bemerkenswert ist, dass sich beide Preisanstiege zeitlich mit den Krim-Blockaden überlappten.

\section{Titanexporte trotz Sanktionen}

Anfang 2015 leitete die russische Regierung eine Verstaatlichungswelle auf der Krim ein, bei der ukrainische Oligarchen wie Rinat Achmetow, Ihor Kolomoisky und Serhiy Taruta ihre Krim-Vermögen verloren haben. Dmytro Firtasch war von der Welle allerdings nicht betroffen. Dem Oligarchen gehören auf der Krim zwei große Chemieunternehmen - die Krim-Soda-Fabrik und Krim-Titan. Dabei hat die Krim-Soda-Fabrik nach der Annexion der Krim viel weniger Probleme als KrimTitan, das von Rohstofflieferungen vom ukrainischen Festland abhängig ist.

Dass es Firtasch gelungen ist, die neue Lieferkette für Krim-Titan erfolgreich zu organisieren, kann man an einer Mitteilung der Group DF ablesen. So lag die Herstellung von Titandioxid durch Krim-Titan im Jahr 2014 auf dem Vorjahresniveau (s. Tabelle 4 auf S. 11), wobei die gesamten Titandioxidexporte im letzten Jahr nur um ein Drittel zurückgingen. Um das Geschäft mit Titandioxid auch nach der Krim-Annexion am Laufen zu halten, musste Firtasch das Unternehmen sowohl nach ukrainischem als auch nach russischem Recht registrieren lassen. Im Juni 2014 wurde deshalb das Unternehmen Titan-Investitionen in Moskau gegründet, das seit Oktober 2014 die Krim-Filiale von dem ukrainischen Unternehmen Krim-Titan pachtet. Im März 2015 wurde Krim-Titan in Ukrainian Chemical Products umbenannt, angeblich um die Sanktionen zu umgehen und Exporte von Titandioxid von der Krim nach Europa zu erleichtern.

Die Versorgung von Krim-Titan mit ukrainischen Rohstoffen sah konkret so aus: Die ukrainische Eisenbahngesellschaft Ukrsalisnyzja, die seit diesem Jahr kei- nen Güterverkehr mehr zwischen dem ukrainischen Festland und der Halbinsel Krim durchführt, hat die Rohstoffe bis zur Grenze in die Region Cherson transportiert. Danach wurden sie über eine private Eisenbahnlinie direkt an Krim-Titan überführt. Genau diesen Punkt haben die Aktivisten im September 2015 blockiert. Aufgrund der Blockade wurden die Rohstofflieferungen aus der Ukraine eingestellt.

Neben der Neuorganisation der Lieferkette musste sich Firtasch auch um die Rohstoffversorgung kümmern. Die Rohstoffbasis der Titanindustrie in der Ukraine bilden in der Region Schytomyr die Kombinate Irschansk, Meschduretschensk und Walky Ilmenit und in der Region Dnipropetrowsk das Kombinat Wilnohirsk. Alle Unternehmen standen bis vor kurzem unter der Kontrolle Firtaschs. Zwei von ihnen die Kombinate Irschansk und Wilnohirsk - hat der Krim-Titan seit 2004 verpachtet. Nach dem Ablauf des Pachtvertrags im September 2014 wurden die Kombinate aber wieder unter staatliche Kontrolle gebracht und werden seitdem von dem Staatsunternehmen Vereintes Bergbau-Chemie-Unternehmen (OGChK) verwaltet. Nach einigen Angaben wird das relativ neue OGChK von dem Oligarchen Ihor Kolomoisky und dem Volksfront-Abgeordneten Mykola Martynenko kontrolliert.

Es ist unklar, ob die Kombinate Irschansk und Wilnohirsk nach dem Wechsel des Verwalters ihre Produktion weiterhin in der Krim verkauft haben oder nicht. Festzuhalten ist aber, dass die zwei anderen Kombinate - Meschduretschensk und Walky Ilmenit - ihre Produktionsvolumen im Jahr 2014 wesentlich erhöht haben (dementsprechend um 200\% und 30\%). Das Produktionswachstum dieser Unternehmen setzte sich auch in diesem Jahr fort. Momentan sieht es aber so aus, als ob OGChK und Group DF ihren Konflikt um die Kombinate beendet haben. Kurz vor dem Beginn der Krim-Blockade hat das OGChK zwei Lieferverträge unterzeichnet, mit dem Saporoshjer Titan-Magnesium-Werk und mit Sumychimprom. Das erste Unternehmen gehört Firtasch zu 49\%, während das zweite von einem loyal zu Firtasch stehenden Manager geleitet wird. Nach der Etablierung der Zusammenarbeit mit OGChK wird Firtasch mit Rohstoffen für seine Titanunternehmen versorgt. Es bleibt dann »nur« noch, den Lieferpunkt in Cherson zu entsperren.

\section{Fazit}

Wie so oft in der Ukraine, klaffen Rhetorik und Realität auch bei der Krim-Frage weit auseinander. Auf der einen Seite fordert die Ukraine, die internationalen Sanktionen gegen Russland wegen der Annexion der Krim zu verschärfen. Auf der anderen Seite führt das Land die Handelsbeziehungen mit der Krim weiter. Der Spagat 
ist schwierig. Einerseits scheinen die Vorwürfe der Krimtataren und rechtsradikaler Organisationen berechtigt zu sein. Anderseits wird in der Ukraine argumentiert, dass man die Menschen auf der Krim, die die Ukraine immer noch als Bürger der Ukraine ansieht, nicht im Stich lassen dürfe. Aus dieser Logik ist eine weitere inkonsequente Politik entstanden, die viele Spielräume bietet, auch für Korruption.

Seit der Annexion der Krim versucht die Ukraine, die Abhängigkeiten der Krim vom ukrainischen Festland geschickt auszunutzen. Außerdem zeigt die Krim-
Blockade deutlich, dass paramilitärische Organisationen in der Ukraine inoffiziell die Strafverfolgungsfunktionen ausüben, während die Kooptation der krimtatarischen Anführer durch Präsident Poroschenko diese zu einem Instrument seiner Außenpolitik werden lässt. Auf eine der Forderungen der Krimtataren - die Abschaffung der Krim-Wirtschaftszone - wird die ukrainische Regierung aber eher nicht eingehen. Denn die ukrainischen Behörden stellen langsam auf die neue Realität um, die eng mit Wirtschaftsinteressen verbunden ist.

\section{Über die Autorin:}

Katerina Bosko ist Wissenschaftliche Mitarbeiterin an der Forschungsstelle Osteuropa an der Universität Bremen und Redakteurin der Online-Zeitschrift »Ukraine-Analysen«. Zugleich schreibt sie ihre Doktorarbeit über die Instrumentalisierung der Diskurse in der ukrainischen Gaspolitik.

\section{Ausgewählte Daten zu den Handelsbeziehungen der Ukraine mit der Krim}

\section{Tabelle 1: Ausgewählte Lebensmittelpreise in der Ukraine und auf der Krim im September 2015}

\begin{tabular}{|l|c|c|c|c|}
\hline \multirow{2}{*}{ Lebensmittel } & \multicolumn{2}{|c|}{ Krim } & $\begin{array}{c}\text { Ukraine, } \\
\text { ukrainische } \\
\text { Hrywnja }\end{array}$ & Preisunterschied, \% \\
\cline { 2 - 3 } Kartoffeln & russische Rubel & ukrainische Hrywnja & 4,9 & 79,9 \\
Schweinefleisch & 27,2 & 8,9 & 77,1 & 21,7 \\
Rindfleisch & 287,7 & 93,8 & 84,6 & 18,9 \\
Zucker & 308,3 & 100,5 & 11,7 & 54,7 \\
Karotten & 55,7 & 18,2 & 8,0 & 28,6 \\
Nudeln & 31,6 & 10,3 & 9,3 & 20,2 \\
Reis & 67,3 & 21,9 & 17,8 & 25,4 \\
Weizenbrot & 65,8 & 21,5 & 10,0 & 45,9 \\
Milch 2,5\% Fett & 38,6 & 12,6 & 10,9 & 81,1 \\
Sauerrahm & 48,7 & 15,9 & 27,0 & 45,1 \\
Rote Bete & 150,0 & 48,9 & 5,5 & 48,6 \\
Zwiebeln & 24,4 & 8,0 & 5,9 & 36,2 \\
\hline Kohl & 26,7 & 8,7 & 8,2 & 2 \\
\hline
\end{tabular}

Anmerkung: Die Lebensmittelpreise auf der Krim wurden nach dem offiziellen Kurs der Nationalen Bank der Ukraine für September 2015 (0,326 Hrywnja/Rubel) umgerechnet.

Quelle: Berechnungen von Katerina Bosko nach Angaben der russischen und ukrainischen Statistikämter, <http://crimea.gks.ru/wps/ wcm/connect/rosstat_ts/crimea/resources/e112e1804a1f4e4fb7e8ff8bfb59418c/\%D1\%86\%D0\%B5\%D0\%BD\%D1\%8B+\%D1 $\% 80 \% \mathrm{D} 0 \% \mathrm{~B} 0 \% \mathrm{D} 0 \% \mathrm{~B} 9 \% \mathrm{D} 0 \% \mathrm{BE} \% \mathrm{D} 0 \% \mathrm{BD} \% \mathrm{D} 0 \% \mathrm{BE} \% \mathrm{D} 0 \% \mathrm{~B} 2 . \mathrm{pdf}>$, <http://www.ukrstat.gov.ua/operativ/operativ2015/ct/ mon_c/mon_c_09_2015/mon_c_30_09_2015.zip> 
Tabelle 2: Warenhandel der Krim mit der Ukraine nach Angaben der russischen Behörden, Millionen US-Dollar

\begin{tabular}{|l|c|c|c|c|}
\hline & $\begin{array}{c}\text { April-Dezember } \\
\mathbf{2 0 1 4}\end{array}$ & $\%$ & Januar-August & 2015 \\
Export aus der Krim & 3,5 & 2,4 & 17,5 & 28,5 \\
Import in die Krim & 2,6 & 3,1 & 36,4 & 45,3 \\
Warenhandel & 6,1 & 2,6 & 53,8 & 38,0 \\
\hline
\end{tabular}

Quelle: Berechnungen von Katerina Bosko nach Angaben für April bis Dezember 2014 - <http://gosstat.crimea.ru/2010/operativ/ arhukoves3-2014.php>, für Januar bis August 2015 - <http://crimea.gks.ru/wps/wcm/connect/rosstat_ts/crimea/resources/c718f9 804a3ae4dab500fd758571d025/\%D0\%BE\%D1\%84.\%D1\%81\%D1\%82\%D0\%B0\%D1\%82+\%D1\%81\%D0\%B0\%D0\%B9\%D $1 \% 82+\%$ D0\%92\%D0\%AD\%D0\%A1+\%D0\%B3\%D0\%B5\%D0\%BE+08.pdf>

\section{Grafik 1: Stromimport der Ukraine aus Russland in den Jahren 2007-2015}

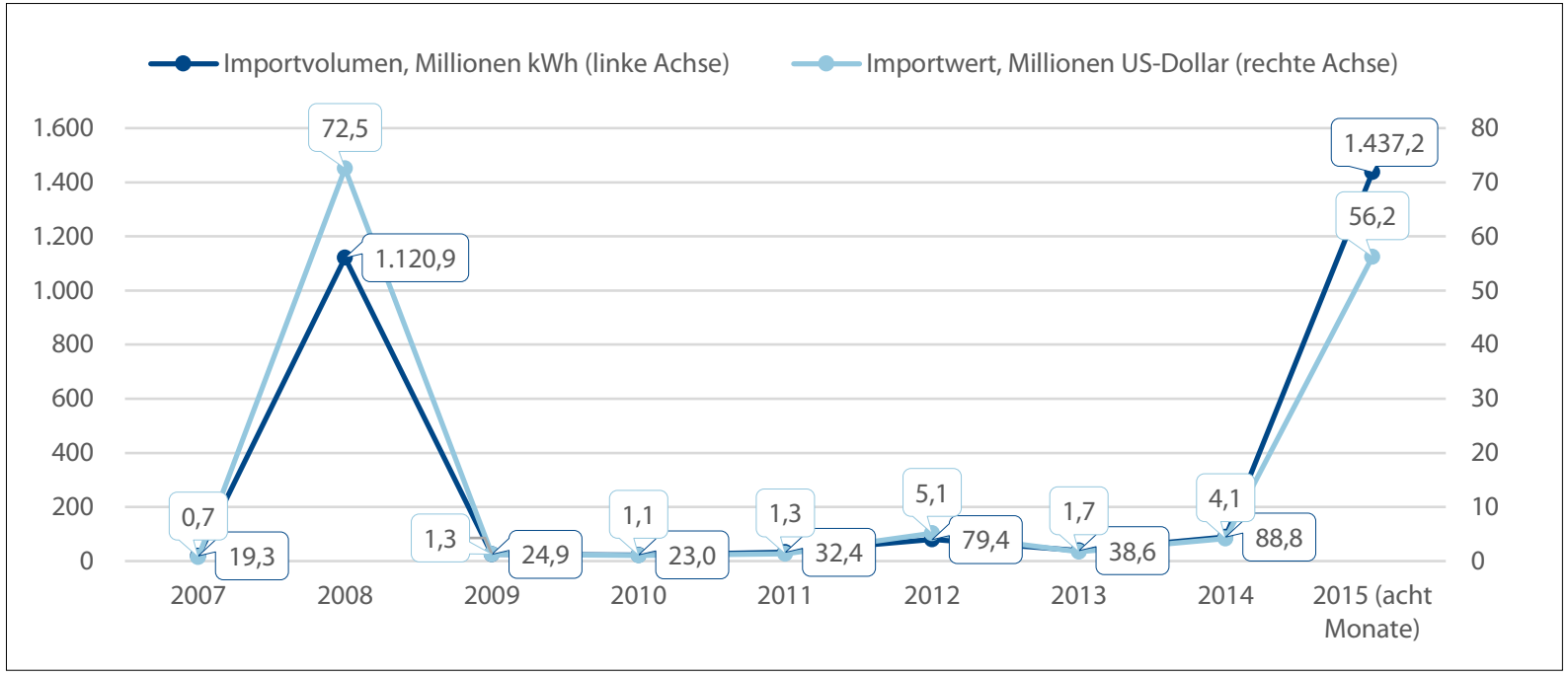

Quelle: Staatliches Statistikamt der Ukraine. Zusammenstellung von Katerina Bosko.

Tabelle 3: Stromlieferungen der Ukraine in die Krim 2014 und Schätzungen für 2015

Exporte von der Ukraine in die Krim, Milliarden $\mathrm{kWh}$

Strompreis für Exporte der Ukraine in die Krim, Rubel/kWh

Exportwert in die Krim, Milliarden Rubel

Importe aus Russland, Milliarden kWh

Strompreis für Importe aus Russland, Rubel/kWh

Importwert aus Russland, Milliarden Rubel

Bilanz für die Ukraine, Milliarden Rubel

* Preis Anfang 2015 laut Medienberichten

** Schätzung; Verdopplung des Werts für das erste Halbjahr 2015 (1,34 Milliarden kWh nach Angaben des ukrainischen Statistikamtes)

Quelle: Berechnungen von Katerina Bosko.
$2015 \mathrm{f}$

6,60

2,99 *

19,73

$2,68^{* *}$

2,4 *

12,87

6,86 
Tabelle 4: Ukrainische Produktion und ukrainischer Exporte von Titandioxid in den Jahren 2013 und 2014 und den ersten acht Monaten im Jahr 2015

\begin{tabular}{|l|c|c|c|}
\hline & $\mathbf{2 0 1 3}$ & $\mathbf{2 0 1 4}$ & $\mathbf{2 0 1 5}$ (acht Monate) \\
Produktion, Tausend Tonnen * & 108 & 101 & k. A. \\
Gesamtexporte & \multicolumn{3}{|c|}{} \\
$\quad$ Tausend Tonnen & 131,8 & 97,2 & 22,9 \\
Millionen US-Dollar & 285,0 & 196,2 & 34,0 \\
\hline
\end{tabular}

* durch Ukrainian Chemical Products (ehemals Krim-Titan)

Quelle: Daten für die Produktion laut Mitteilungen der Group DF(<https://groupdf.com/uk/pres-tsentr/novini/krimskiy-titan-zbils hiv-virobnitstvo-dioksidu-titanu-ta-sirchanoyi-kisloti-u-pershomu-kvartali-2014-roku/; https://groupdf.com/uk/pres-tsentr/novi ni/u-2014-rotsi-krimskiy-titan-zberig-obsyagi-virobnitstva-osnovnoyi-produktsiyi-dioksidu-titanu/>), Exportdaten nach Angaben des Staatlichen Statistikamts der Ukraine. Zusammenfassung von Katerina Bosko.

Tabelle 5: Titandioxidexporte aus der Ukraine in einige Länder im Jahr 2014 und den ersten acht Monaten 2015

\begin{tabular}{|l|c|c|c|c|}
\hline \multirow{2}{*}{} & \multicolumn{2}{|c|}{2014} & \multicolumn{2}{c|}{2015 (acht Monate) } \\
\cline { 2 - 5 } & $\begin{array}{c}\text { Millionen } \\
\text { US-Dollar }\end{array}$ & $\%$ & $\begin{array}{c}\text { Millionen } \\
\text { US-Dollar }\end{array}$ & $\%$ \\
Russland & 43,4 & 22,1 & 14,4 & 26,9 \\
Deutschland & 50,1 & 25,5 & 9,1 & - \\
\hline Italien & 20,9 & 10,7 & - & 4,7 \\
USA & 9,6 & 4,9 & 1,6 & 4,8 \\
Kanada & 8,3 & 4,2 & 1,6 & 2,0 \\
Frankreich & 6,4 & 3,3 & 0,7 & 0,1 \\
Spanien & 6,3 & 3,2 & 0,03 & 3,9 \\
Andere & 5,4 & 2,8 & 1,3 & 15,2 \\
Gesamt & 45,8 & 23,4 & 5,2 & 100 \\
\hline
\end{tabular}

Quelle: Staatliches Statistikamt der Ukraine. Zusammenstellung von Katerina Bosko. 


\section{Demographische Situation auf der Krim: aktuell und im Rückblick}

Tabelle 1: Bevölkerungsgröße auf der Halbinsel Krim nach Nationalitäten gemäß Volkszählungen in verschiedenen Jahren, Tausend Personen

\begin{tabular}{|l|c|c|c|c|c|}
\hline & Russen & Ukrainer & Krimtataren* & Andere & Gesamt \\
\hline 1926 & 143,3 & 40,9 & 89,2 & 72,2 & 345,7 \\
1939 & 558,5 & 154,1 & 218,9 & 194,9 & $1.126,4$ \\
1959 & 857,9 & 267,9 & 0,4 & 75,3 & $1.201,5$ \\
1970 & $1.220,5$ & 480,7 & 2,1 & 110,2 & $1.813,5$ \\
1989 & $1.461,0$ & 547,3 & 5,4 & 122,2 & $2.135,9$ \\
2001 & $1.629,5$ & 625,9 & 38,4 & 136,7 & $2.430,5$ \\
2014 & $1.450,4$ & 576,6 & 245,3 & 128,9 & $2.401,2$ \\
\hline
\end{tabular}

* Angaben vor 1959 ohne Unterscheidung in Krimtataren und Tataren. Danach nur für Krimtataren.

Quelle: Volkszählungen der Sowjetunion laut Institut für Demographie an der Nationalen Forschungsuniversität Higher School of Economics (<http://demoscope.ru/weekly/ssp/rus_nac_26.php?reg=788>; <http://demoscope.ru/weekly/ssp/rus_nac_39.php?reg=68>; $<$ http://demoscope.ru/weekly/ssp/resp_nac_59.php?reg=9>; $<$ http://demoscope.ru/weekly/ssp/resp_nac_70.php? reg=12>; $<$ http:// demoscope.ru/weekly/ssp/resp_nac_79.php?reg=12>; <http://demoscope.ru/weekly/ssp/resp_nac_89.php?reg=11>; <http://demo scope.ru/weekly/2003/0113/analit03.php>), die Volkszählung in der Republik Krim im Jahr 2014, <http://crimea.gks.ru/wps/wcm/ connect/rosstat_ts/crimea/resources/1f72198049859f4b9205f22d12c3261e/pub-04-01.pdf>. Zusammenfassung der Redaktion der Ukraine-Analysen.

Grafik 1: Bevölkerungsstruktur auf der Halbinsel Krim nach Nationalitäten gemäß Volkszählungen in verschiedenen Jahren, \%

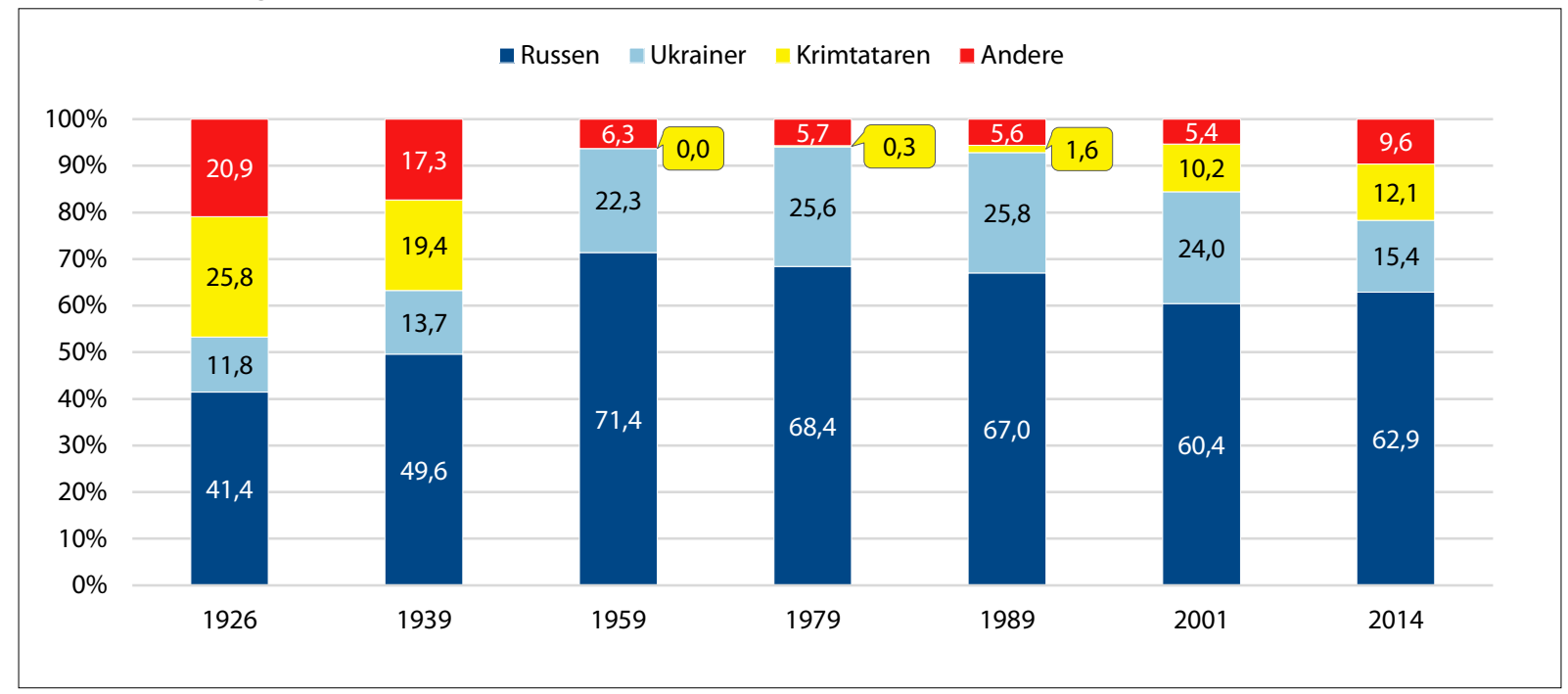

Quelle: Volkszählungen der Sowjetunion laut Institut für Demographie an der Nationalen Forschungsuniversität Higher School of Economics (<http://demoscope.ru/weekly/ssp/rus_nac_26.php?reg=788>; <http://demoscope.ru/weekly/ssp/rus_nac_39.php?reg=68>; $<$ http://demoscope.ru/weekly/ssp/resp_nac_59.php?reg=9>; <http://demoscope.ru/weekly/ssp/resp_nac_70.php?reg=12>; <http:// demoscope.ru/weekly/ssp/resp_nac_79.php?reg=12>; <http://demoscope.ru/weekly/ssp/resp_nac_89.php?reg=11>; <http://demo scope.ru/weekly/2003/0113/analit03.php>), die Volkszählung in der Republik Krim im Jahr 2014, <http://crimea.gks.ru/wps/wcm/ connect/rosstat_ts/crimea/resources/1f72198049859f4b9205f22d12c3261e/pub-04-01.pdf>. Zusammenfassung der Redaktion der Ukraine-Analysen. 
Tabelle 2: Bevölkerungsgröße auf der Halbinsel Krim nach Muttersprachen gemäß Volkszählung im Jahr 2014

\begin{tabular}{|c|c|c|c|c|}
\hline & & \multicolumn{3}{|c|}{ Muttersprache } \\
\hline & & Russisch & Ukrainisch & $\begin{array}{c}\text { Krim-Tatarisch oder } \\
\text { Tatarisch }\end{array}$ \\
\hline \multirow{5}{*}{ 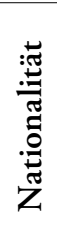 } & Russen & 1.184 .321 & 1.615 & 180 \\
\hline & Ukrainer & 228.685 & 62.115 & 74 \\
\hline & Krimtataren & 12.502 & 53 & 216.149 \\
\hline & Andere & 58.592 & 524 & 33.569 \\
\hline & Gesamt* & 1.484 .100 & 64.307 & 249.972 \\
\hline
\end{tabular}

* Gesamtzahl derjenigen, die sowohl Nationalität als auch Muttersprache angegeben haben.

Quelle: Volkszählung in der Republik Krim im Jahr 2014, <http://crimea.gks.ru/wps/wcm/connect/rosstat_ts/crimea/resources/f74 a898049859bdd9180f12d12c3261e/pub-04-06.pdf>. Zusammenfassung der Redaktion der Ukraine-Analysen.

Tabelle 3: Bevölkerungsgröße auf der Halbinsel Krim nach Muttersprachen gemäß Volkszählungen in den Jahren 1897 und 2014, Tausend Personen

\begin{tabular}{|l|c|c|c|c|c|}
\hline & Russisch & Ukrainisch & $\begin{array}{c}\text { Krim-Tatarisch } \\
\text { oder Tatarisch }\end{array}$ & Andere & Gesamt \\
\hline 1897 & 180,96 & 64,70 & 194,29 & 106,63 & 546,59 \\
2015 & 1484,10 & 64,31 & 249,97 & 21,06 & 1819,43 \\
\hline
\end{tabular}

Anmerkung: Die Zahlen für 1897 beziehen sich ausschließlich auf die Halbinsel Krim (d. h. das Gouvernement Taurien abzüglich der Angaben für die Kreise Berdjansk, Dnepr und Melitopol).

Quelle: Erste allgemeine Volkszählung des Russischen Reichs im Jahr 1897, <http://demoscope.ru/weekly/ssp/rus_lan_97_uezd. php?reg=1420>; Volkszählung in der Republik Krim im Jahr 2014, <http://crimea.gks.ru/wps/wcm/connect/rosstat_ts/crimea/resour ces/f74a898049859bdd9180f12d12c3261e/pub-04-06.pdf>. Zusammenfassung der Redaktion der Ukraine-Analysen.

Grafik 2: Bevölkerungsgröße auf der Halbinsel Krim nach Muttersprachen gemäß Volkszählungen in den Jahren 1897 und 2014 (\%)

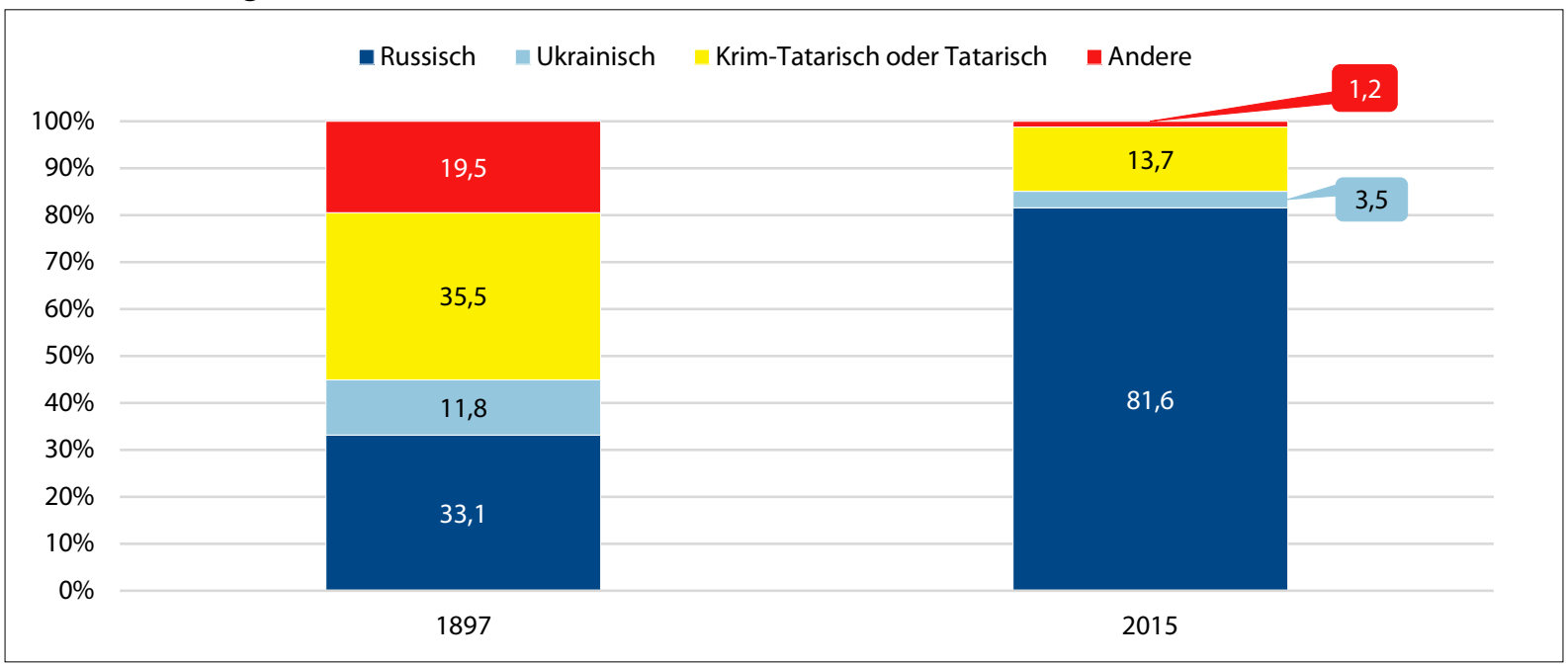

Anmerkung: Die Zahlen für 1897 beziehen sich ausschließlich auf die Halbinsel Krim (d. h. das Gouvernement Taurien abzüglich der Angaben für die Kreise Berdjansk, Dnepr und Melitopol).

Quelle: Erste allgemeine Volkszählung des Russischen Reichs im Jahr 1897, <http://demoscope.ru/weekly/ssp/rus_lan_97_uezd. php?reg=1420>; Volkszählung in der Republik Krim im Jahr 2014, <http://crimea.gks.ru/wps/wcm/connect/rosstat_ts/crimea/resour ces/f74a898049859bdd9180f12d12c3261e/pub-04-06.pdf>. Zusammenfassung der Redaktion der Ukraine-Analysen. 


\section{Die Lage der Menschenrechte auf der Krim}

\section{Report of the Human Rights Assessment Mission on Crimea (6-18 July 2015), 17 September 2015}

\section{Executive Summary ${ }^{1}$}

1. Following an invitation by the Government of Ukraine on 15 June 2015, the OSCE Office for Democratic Institutions and Human Rights (ODIHR) and the OSCE High Commissioner on National Minorities (HCNM) conducted a joint Human Rights Assessment Mission (HRAM) on Crimea from 6 to 18 July 2015.

2. The HRAM evaluated the current human rights situation in Crimea, including the situation of minority groups, as impacted by developments since the release of the previous ODIHR/HCNM report on Ukraine in May 2014, soon after the occupation and annexation of Crimea by the Russian Federation.

3. Notably, the most critical human rights problems in Crimea today are largely congruent with the concerns and negative trends identified in that previous assessment, which ODIHR and HCNM then called upon de facto authorities in Crimea to address.

4. Despite their clear mandates to monitor the human rights situation in Crimea, the institutions and independent experts of the OSCE, the United Nations and the Council of Europe have all had their access to the Crimean peninsula either fully or partially restricted since the annexation. The de facto authorities in Crimea did not respond to requests to facilitate access to Crimea for the HRAM, for which reason the HRAM primarily conducted fact-finding and research in the territory of mainland Ukraine, as well as through remote interviews with relevant contacts in Crimea and elsewhere.

5. Through extensive meetings and interviews with over 100 civil society actors, Ukrainian authorities, internally displaced persons and cross-boundary travellers, the HRAM received numerous credible, consistent and compelling accounts of human rights violations and legal irregularities in Crimea-some of them of a serious nature. The allegations documented and trends established by the HRAM demand urgently to be addressed by Crimean de facto authorities, and underscore the need for systematic independent monitoring of the human rights situation in Crimea by impartial international bodies.

6. As a result of the annexation, the changes in government and the legal framework being applied in Crimea have dramatically impacted the enjoyment of the full spectrum of human rights and fundamental freedoms by residents there, particularly of those residents who were opposed to the annexation, were unable to reject forced Russian citizenship, and/or did not seek to acquire Russian passports.

7. Fundamental freedoms of assembly, association, movement, expression and access to information have all been restricted in some fashion-whether through formal measures, or through the sporadic targeting of individuals or communities representing opposing views, voices or socio-political structures.

8. Re-registration requirements by the Russian Federation for non-governmental organizations (NGOs), media outlets, and religious organizations have reportedly been leveraged against those opposed to Russian rule, significantly restricting freedom of association, constricting the space for civil society, and decimating the number of independent voices in the media landscape.

9. Through the justice system, the de facto authorities in Crimea have applied vague charges of "extremism" and "separatism" under criminal law of the Russian Federation to a wide variety of assemblies, speech and activities-in some cases retroactively to events prior to annexation and/or outside of Crimea in mainland Ukraine. Based on interviews with those targeted and primary documentation reviewed by the HRAM, numerous such criminal warnings, investigations and prosecutions appeared to be politically motivated-directed at pro-Ukrainian activists, journalists and minority community members-without due process guarantees for the accused, and without effective remedies for alleged procedural violations.

10. In contrast, there appear to have been neither proactive investigations nor any prosecutions of pro-Russian "selfdefence" groups accused of committing serious human rights abuses at the start of and since the occupation of Crimea. Those alleged abuses included disappearances, extrajudicial killings, torture and ill-treatment, as documented by 
ODIHR and HCNM in their 2014 joint HRAM report. Since then, "self-defence" groups have reportedly continued to intimidate, harass, detain and seize the properties of Crimean residents—-particularly those suspected of opposing Russian rule-without an adequate legal basis.

11. In terms of accountability, the European Court of Human Rights has extended the Russian Federation's deadline until 25 September 2015 to submit its observations on the admissibility of two inter-State applications lodged against it by Ukraine-including in relation to forced citizenship, discrimination, property rights, the right to private life, and the prohibition against torture and ill-treatment. During that extended response period, the Constitutional Court of the Russian Federation issued a concerning ruling on 14 July 2015 that the government would not be required to implement judgments of the European Court of Human Rights if they contravened the Russian Constitution. Apparently conflicting with the Russian Federation's obligations under international treaty law, such a decision could further undermine the right to an effective remedy for claimants, and the execution of judgments by the European Court in future claims, including in the dozens of individual cases that have already been submitted to the Court in relation to recent events in Crimea.

12. In the realm of economic, social and cultural rights, the imposition of Russian Federation citizenship and laws on residents of Crimea has caused problems for those Ukrainian citizens who have not sought Russian passports (despite having Russian citizenship nominally imposed upon them). Without Russian passports, residents face obstacles in every aspect of their lives, including: re-registering and/or selling private properties and businesses; gaining or retaining employment; and accessing education, health care, or other social services. Language studies and native-language education in the Ukrainian and Crimean Tatar languages have also reportedly been reduced in schools and universities throughout Crimea, to the detriment of those communities' enjoyment of their cultural and language rights.

13. In the penitentiary system, more than 2,000 convicts imprisoned in Crimea at the time of annexation reportedly were unable to opt out of mandatory Russian citizenship, did not benefit from Ukrainian-ordered amnesties and conditional releases in 2014, and are potentially subject to transfer to penal colonies in mainland Russia, as has reportedly transpired in some cases. Injecting drug users and persons living with HIV/AIDS in the pre-trial detention facility and three penal colonies in Crimea have also reportedly lacked necessary medical care.

14. Exacerbating the legal and practical problems enumerated above are the existence of dual and parallel citizenship records, civil registries, cadastral records, pension systems, and justice systems exercising jurisdiction over the same persons and properties. As neither Russia nor Ukraine recognizes official documentation issued by the other in relation to Crimea, residents are caught between two overlapping and conflicting legal and regulatory systems. In order to navigate these complexities, many Crimean residents keep both Russian and Ukrainian passports, despite both countries not recognizing those residents' dual citizenship of the other.

15. The HRAM received numerous accounts of Crimean residents and displaced persons who were unable to: sell their properties or businesses; acquire Ukrainian birth certificates for newly born children; or have their divorces in Crimea acknowledged by Ukrainian authorities, resulting in restrictions of the freedom of movement of many children with single parents under new Ukrainian regulations on travel to Crimea. Students graduating from Crimean secondary schools since annexation have also been unable to enroll in Ukrainian universities with diplomas issued by unrecognized authorities (and without sufficient opportunities to seek alternative qualifications), spurring surges in migration of families with school-age children from Crimea to mainland Ukraine.

16. The HRAM found in Crimea that those Crimean Tatars and Ukrainians who openly supported the territorial integrity of Ukraine and did not support the de facto authorities continued to be in a particularly vulnerable position. The suppression of activities of Mejlis—a self-governing body of Crimean Tatars—as well as intimidation, expulsion, or incarceration of prominent leaders of the Mejlis of the Crimean Tatar People has had a detrimental effect on the exercise of political and civil rights of persons belonging to the Crimean Tatar community.

17. Effectively forcing Crimean Tatar community-run media outlets, such as ATR, to close by denying their registration has not only restricted media freedom and access to information, but also deprived the Crimean Tatar community of a vital instrument to maintain and revitalize its identity.

18. The space for Ukrainian culture in the illegally annexed Crimea has also decreased. Cultural, religious and symbolic elements of Ukrainian identity have been restricted and/or suppressed through various administrative or lawenforcement measures. Hostile attitudes in Crimea towards residents of Crimea who support the territorial integrity of Ukraine, display Ukrainian state and cultural symbols and publicly celebrate important dates for the Ukrainian culture and history are widespread. 
19. Education in and of the Ukrainian language is disappearing. Pressure on school administrations, teachers, parents and children to discontinue teaching in and of the Ukrainian language is growing, which further curtails the presence of the Ukrainian language and culture on the peninsula. Education both in and of the Crimean Tatar language continues to face obstacles.

20. As obligated under its international human rights commitments and the Constitution of Ukraine, the Ukrainian government has adopted numerous policy measures to meet the needs of its citizens remaining in, or displaced from Crimea, despite lacking effective control over the peninsula. Those measures have reportedly been most effective where conjoined with awareness-raising campaigns to inform affected populations of solutions available for the challenges they face. However, many of those citizens impacted by the political and security challenges in Crimea over the last year have called for more relief and administrative assistance from the Ukrainian government to overcome those problems - particularly in relation to accessing the civil registry and education, and acquiring personal identification or other official documents. People crossing back and forth between Crimea and mainland Ukraine have also criticized newly increased restrictions on freedom of movement between the two regions, and inadequate infrastructure at land crossing points.

21. Through the Crimean de facto authorities, the Russian Federation is likewise obligated to respect, protect and fulfil the human rights and fundamental freedoms of persons in Crimea-in line with the international treaties to which it is party, as well as its commitments as an OSCE participating State to uphold those human rights and fundamental freedoms. Those OSCE commitments encompass the Russian Federation's obligations under international human rights law and international humanitarian law, per its role as an occupying power in effective control of the Crimean peninsula.

Quelle: <http://www.osce.org/odihr/180596?download=true>

\section{Die Krim-Blockade in den Berichten der OSZE-Beobachtermission in der Ukraine (Auszüge)}

\section{September 2015}

On 20 September, in Kherson region the SMM monitored blocking of three crossing points to Crimea, namely Kalanchak, Chaplynka and Chonhar organized by the Crimean Tatars' self-governing body, the Mejlis. While no trucks were allowed to pass, passenger vehicles and pedestrian traffic were allowed through in both directions at all three crossing points. The situation was occasionally tense but there were no incidents. In Kalanchak $(95 \mathrm{~km}$ south-east of Kherson) the SMM observed uniformed members of the Right Sector and Sich Cossacks along with Crimean Tatars. The Security Service of Ukraine (SBU), regular police, Kherson volunteer police battalion and special police forces were also present. In Kalanchak, there were tensions between protesters and truck drivers after the protesters accused drivers of supporting the Russian army. Truck drivers claimed that the protesters threatened to set their trucks on fire if they did not turn back. The head of the Crimean Tatars' self-governing body, Mr. Chubarov visited the gathering and told the SMM that the blockade would continue indefinitely.

Ten kilometres north of Chaplynka crossing point ( $90 \mathrm{~km}$ south-east of Kherson), the SMM observed Crimean Tatars and approximately 25 members of the Right Sector and 50 Sich Cossacks in camouflage clothes. Six regular police officers and 20-25 Kherson police battalion volunteers were present. A group of 20 civilians demonstrated peacefully. In Chonhar (162km south-east of Kherson) the roadblock was manned by approximately 50 members of the Right Sector, Maidan Self-Defence and Aidar volunteer battalion under the Ministry of Defence and Donbas volunteer battalion under the Ministry of Internal Affairs. Twenty members of Kherson volunteer police battalion were present. Crimean Tatar representatives blocked the road by walking in circles over the pedestrian road crossing. The SMM observed two empty trucks (with Donetsk and Zaporizhzhia license plates) being stopped by the protesters and prevented from proceeding towards the mainland. The protesters threatened to slash the trucks' tyres if trucks do not turn back. At the end of its observation, the SMM observed several empty trucks pass the blockade towards Ukraine mainland.

Quelle: <http://www.osce.org/ukraine-smm/183736> 


\section{September 2015}

At the three crossing points to Crimea-Kalanchak, Chaplynka and Chonhar (95, 90 and 162km south-east of Kherson, respectively) — the SMM observed a calm situation. The SMM observed fewer cargo trucks queuing at Kalanchak and Chaplynka than on previous days. At Kalanchak, the SMM also observed that about 20 protesters, including Right Sector and Avtomaidan members, Crimean Tatars and a man bearing Chechen insignia, were stopping and searching private vehicles.

Quelle: <http://www.osce.org/ukraine-smm/184821>

\section{September 2015}

The SMM continued to monitor the situation at the three crossing points to Crimea-Kalanchak, Chaplynka, and Chonhar (95, 90 and 162 km south-east of Kherson, respectively) — which was relatively calm and stable. The head of Kalanchak border guards unit told the SMM that an informal co-ordination committee, consisting of the Kherson regional state administration and the local law enforcement agencies assessed on 23 September the practice of protesters checking the trunks of the passenger vehicles and travellers' belongings (see the SMM Daily Report 24 September 2015) as "wrongful and unlawful". The SMM did not observe law enforcement preventing protesters from searching vehicles. The SMM observed that protesters continued to check passenger cars on a random basis at the Kalanchak crossing point, even though most of the vehicles could travel unhindered.

Quelle: <http://www.osce.org/ukraine-smm/185676>

\section{September 2015}

In Kherson, the SMM followed up on media reports about damage caused by an explosion at the Office of the Representative of the President of Ukraine in Crimea. The SMM saw a damaged wooden door and a broken window located over the entrance door as well as a small amount of debris. The SMM saw a group of police forensics specialists on site conducting investigation of the premises. A police officer told the SMM that a small explosive device detonated at the doors to the Office around 3:00hrs on 30 September. The police officer said that the investigation department of the Security Service of Ukraine in Kherson region has taken over the investigation.

Quelle: <http://www.osce.org/ukraine-smm/188651>

\section{October 2015}

Near the Kalanchak checkpoint at the Administrative Boundary Line to Crimea (95km south-east of Kherson), the SMM saw five members of the Aidar volunteer battalion under the Ministry of Internal Affairs (MIA), the Right Sector, and several Crimean Tatars, checking cars going to and from Crimea. In an area $6 \mathrm{~km}$ north of the Kalanchak checkpoint, the SMM saw two road blocks, each manned by five or six men wearing camouflage fatigues, some wearing the insignia of Right Sector, Odessa Avtomaidan, Self-defence and the Union of Anti-Terrorist Operation Veterans. Approximately 25 activists from the aforementioned groups (mainly men) were in the vicinity. Nearby, the SMM observed the construction of a checkpoint, supervised by armed men who identified themselves as members of the Kherson volunteer battalion under the MIA.

Quelle: <http://www.osce.org/ukraine-smm/189231>

\section{October 2015}

At the Kalanchak and Chaplynka crossing points (77 and 70km south-east of Kherson, respectively) on the administrative boundary line with Crimea, district police and the border guard shift commander, respectively, told the SMM on 7 October that many drivers passing through the checkpoints had both officially and unofficially complained about activists searching their vehicles. Activists at both crossing points told the SMM that drivers generally agreed to have their vehicles searched. The border guard shift commander at Kalanchak told the SMM on 7 October that four drivers attempting to cross the administrative boundary line had the previous day refused to allow activists to search their vehicles. He said in one instance, activists had smashed the rear window of one car. A Right Sector member confirmed the allegation to the SMM but claimed the action had come after the car had driven over an activist's foot.

Quelle: <http://www.osce.org/ukraine-smm/191346> 


\section{October 2015}

On 12-13 October the SMM monitored the situation at Chonhar and Kalanchak crossing points $(160 \mathrm{~km}$ and $92 \mathrm{~km}$ south-east of Kherson respectively) at the administrative boundary line with Crimea. The SMM observed activists logging the licence plates of the travelling vehicles into a notebook computer, while an interlocutor had told the SMM that the activists also run these numbers through a computer programme.

The SMM also observed that the law enforcement personnel present at the checkpoints run by activists were actively involved in the joint searches of the passenger vehicles. This reflects a contrast with the situation several days ago (see SMM Daily Report 9 October 2015) when the activists in camouflage clothes alone conducted the vehicle searches. A representative of the Kherson police battalion said that his unit conducted vehicles checks jointly with the civil activists, a traffic police unit, and two police officers from the district headquarters. The SMM witnessed one male driver questioning the legality of the search of his vehicle, which was being conducted jointly by law enforcement and the activists.

A Right Sector (Pravyi Sektor) member told the SMM that similar arrangements on joint vehicle searches by activists and law enforcement personnel were in place at Chaplynka $(70 \mathrm{~km}$ south-east of Kherson) crossing point.

Quelle: <http://www.osce.org/ukraine-smm/192756>

\section{October 2015}

In Kherson the SMM followed up on an incident, which according to Kherson deputy police chief had involved the use of an explosive device in an apparent attempt on 7 October to destroy an electricity supply pylon in the vicinity of Chonhar (160km south-east of Kherson). Next to the remnants of an old pylon the SMM observed a new pylon had been erected, which is a part of the power grid supplying Crimea with electricity. A resident from a neighbouring settlement told the SMM that the explosion had occurred some $2 \mathrm{~km}$ away and its power had caused the glass windows to shake. The interlocutor said that local residents believed the incident was linked to the Crimea "blockade", since before it began the area was quiet. In a conversation with the SMM, a Right Sector (Pravyi Sektor) member participating in the "blockade" stated his group had not been involved.

Quelle: <http://www.osce.org/ukraine-smm/193151>

\section{October 2015}

Near the checkpoint at Chonhar $(162 \mathrm{~km}$, south-east of Kherson), on the Administrative Boundary Line between Crimea and Kherson region, the SMM observed damage and repair works to a pylon supplying electricity to the Crimean peninsula, where an explosion was reported in the early hours. The incident site is approximately $1 \mathrm{~km}$ from the site where another pylon from the same power grid was damaged on 7 October 2015 (see SMM Daily Report 18 October 2015). At the site, the SMM observed the police keeping a security perimeter of some $300 \mathrm{~m}$ as well as an Explosive Ordnance Unit from the Kherson regional department of the Ministry of Internal Affairs (MIA), who removed three $82 \mathrm{~mm}$ mortars, which had been taped to the pylon with detonators positioned in between. Local and national media as well as Crimean Tatars, Azov battalion under the MIA and Right Sector activists were also present at the site.

Quelle: <http://www.osce.org/ukraine-smm/193676> 


\section{Die Rumänen und Ungarn der Ukraine - stille Minderheiten?'}

Von Oleg Friesen, München

\section{Zusammenfassung}

Der Zusammenstoß ukrainischer Sicherheitskräfte mit Kämpfern des Rechten Sektors im Juli dieses Jahres führte zu einem diplomatischen Skandal zwischen der Ukraine und Ungarn. Die Regierung Orban sieht sich als Advokat der ungarischen Minderheit in der Ukraine und beklagt regelmäßig deren Lage. Davon profitiert auch die russische Propaganda. Doch wie steht es um ethnische Minderheiten in der Ukraine tatsächlich?

\section{Einleitung}

Warum es im Juli in Mukatschewe in der West-Ukraine zu einer tödlichen Schießerei zwischen der ukrainischen Polizei und Kämpfern des Rechten Sektors kam, ist noch immer umstritten. Die ukrainische Regierung wirft dem Rechten Sektor kriminelle Machenschaften vor, der Rechte Sektor meint, gegen genau diese vorzugehen. Viele internationale Beobachter und Medien werten den Vorfall jedoch als Machtlosigkeit der ukrainischen Regierung gegenüber den Freiwilligenbataillonen. Freuen konnte sich das russische Staatsfernsehen, welches erneut von einer Bedrohung durch Nationalisten sprach. Obwohl der Rechte Sektor in der politischen Landschaft der Ukraine auffällt, ist seine tatsächliche Macht gering. Bei den Präsidentschafts- und Parlamentswahlen 2014 erzielte die Partei bzw. ihr Kandidat nur etwa ein Prozent. Auch heute würde der Rechte Sektor nach verschiedenen Umfragen nicht die notwendigen fünf Prozent erreichen, um in die Oberste Rada der Ukraine einzuziehen. Dennoch ist der Vorfall in Transkarpatien alarmierend, deuten ihn viele als Synonym für die politische Instabilität der Ukraine. Besonders besorgt zeigte sich der westliche Nachbar Ungarn. In der Region Transkarpatien leben bis zu 150.000 ethnische Ungarn. Während der ungarische Ministerkabinettsvorsitzende und Fidesz-Politiker Lazar Janos erklärte, im Falle einer Eskalation der Lage in Transkarpatien die ethnischen Ungarn in seinem Land als Flüchtlinge aufzunehmen, sprach der Chef des ungarischen Geheimdienstes von in der Ukraine tätigen ungarischen Agenten, welche die ungarische Minderheit schützen und für die Interessen Budapests eintreten würden. Für das ukrainische Außenministerium war eine solche Aussage ein Affront, für den der ungarische Botschafter in Kiew einbestellt wurde.

Tatsächlich belastet das Thema der ungarischen Minderheit schon seit Jahren das Verhältnis zwischen Kiew

1 Dieser Artikel ist erstmalig auf der Website der Friedrich-Naumann-Stiftung als Hintergrund: Ukraine Nr. 62 am 07. Oktober 2015 unter <https://www.freiheit.org/sites/default/files/uplo ads/2015/10/07/20151007hintergrundukraine.pdf $>$ erschienen. Die Redaktion der Ukraine-Analysen dankt für die Erlaubnis zum Nachdruck. und Budapest, wo es besonders im Wahlkampf aktuell ist. Die rechtskonservative Fidesz-Partei des Ministerpräsidenten Viktor Orban beansprucht für sich, die Rechte ethnischer Ungarn in der Ukraine zu verteidigen und fordert gar eine ungarische Autonomie in Transkarpatien. Ähnliche diplomatische Verstimmungen gab es auch zwischen der Ukraine und Rumänien, denn der Südwesten der Ukraine ist auch Heimat einer starken rumänischsprachigen Minderheit. Doch während das Verhältnis zwischen Kiew und Budapest problematisch ist, erleben die Beziehungen zwischen der Ukraine und Rumänien nach dem Maidan einen positiven Höhepunkt. Oft werden vermeintliche Probleme der ungarischen oder rumänischen Minderheit von Budapest oder Bukarest aufgeblasen - für die Vertreter der Minderheit in der Ukraine spielen sie eine geringere Rolle.

\section{Allgemeines zu ethnischen Minderheiten in der Ukraine}

Die Ukraine ist ein multikulturelles Land. Laut der Bevölkerungszählung von 2001 bezeichnen sich 77,8 \% der Bürger als Ukrainer. Russen stellen mit 17,3\% die größte ethnische Minderheit dar. Die ethnische Zuordnung geht aber nicht unbedingt mit der Muttersprache und der tatsächlich im Alltag gesprochenen Sprache einher: Bis zu $30 \%$ der Ukrainer geben Russisch als Muttersprache an. Die Muttersprache ist aber nicht immer die tatsächlich im Alltag gesprochene Sprache. Viele Ukrainer geben Ukrainisch als Muttersprache an, sprechen im Alltag jedoch Russisch oder die als "Surschyk" bekannte Mischsprache aus Ukrainisch und Russisch. Offizielle Zahlen existieren hierfür nicht. Geht man vom Gebrauch der ukrainischen und russischen Sprache aus, besteht sowohl ein Ost-West-Gefälle wie auch ein Unterschied zwischen Stadt und Land. In der Region Luhansk sprechen die Menschen auf dem Land überwiegend Ukrainisch, in der Stadt Russisch. Allerdings haben auch die südwestlichen Regionen der Ukraine Transkarpatien und Tscherniwzi - einen hohen Anteil russischsprachiger Bevölkerung.

Die politischen und kulturellen Rechte der Minderheiten waren seit der Unabhängigkeit der Ukraine 
umstritten, besonders das Recht auf sprachliche Autonomie. Obwohl das ukrainische Recht keine doppelte Staatsbürgerschaft vorsieht, haben viele Bürger nicht-ukrainischer Abstammung einen zweiten Pass. National gesinnte politische Gruppierungen in der Ukraine, aber auch der ehemalige Präsident Wiktor Juschtschenko forderten die Einführung von Ukrainisch als alleiniger Amtssprache auf allen Ebenen der Staatsverwaltung. Unter der Regierung Janukowytsch wurde im Jahr 2012 schließlich die Regel eingeführt, nach der in Regionen und Kreisen, in denen eine Minderheit über 10\% ausmacht, deren Sprache den Status einer Regionalsprache erhält. Dies machte Russisch in neun von 27 Regionen bzw. Verwaltungsgebieten und in einigen Kreisen und Gemeinden weiterer Regionen zur zweiten Amtssprache. Rumänisch und Ungarisch sind aufgrund des für die Region nicht ausreichenden Bevölkerungsanteils lediglich in einigen Kreisen Regionalsprachen. Gegenwärtig soll die Dezentralisierungsreform, eines der größten Reformprojekte der Ukraine nach dem Maidan, die Rechte der Minderheiten sichern, denn Kreise und Kommunen erhalten mehr Kompetenzen zur Selbstverwaltung.

\section{Die Rumänen der Nordbukowina}

Bis zu 400.000 Menschen in der Ukraine sprechen Rumänisch als Muttersprache. Sie wären nach den Russen die zweitgrößte ethnische Minderheit des Landes, doch sie unterteilen sich selbst noch in Rumänen und Moldauer. Moldauer sind mit insgesamt 0,4\% der Gesamtbevölkerung die viertgrößte ethnische Minderheit der Ukraine. Für Eugen Petrasch, Leiter des rumänischen Kulturinstituts Eudoxiu Hurmuzachi in Tscherniwzi, ist die Teilung der Menschen in Rumänen und Moldauer noch eine Folge der sowjetischen Moldauisierungspolitik. Die heutige Region Tscherniwzi, historisch Nordbukowina genannt, gehörte vor 1940 zum Königreich Rumänien und wurde in Folge des Hitler-StalinPaktes von der Roten Armee besetzt und annektiert, so wie Bessarabien. Die Nordbukowina und Südbessarabien kamen mit Verweis auf die dortige ukrainische Bevölkerungsmehrheit zur Ukrainischen Sowjetrepublik und sind heute die ukrainischen Regionen Tscherniwzi und der westliche Teil der Region Odessa. Bessarabien selbst wurde zur Moldauischen Sowjetrepublik, der heutigen Republik Moldau. Die Sichtweise von Eugen Petrasch, dass Moldauer Rumänen seien, hat unter den Rumänisch sprechenden Menschen Befürworter und Gegner. Befürworter sprechen von der Erfindung einer moldauischen Identität durch den Stalinismus, um die gewaltsame Einverleibung der Gebiete und die Schaffung einer Moldauischen Sowjetrepublik zu rechtfertigen. Durch Maßnahmen wie die Einführung eines kyrillischen Alphabets für die Moldauer sowie die »Slawisierung« von rumänisch klingenden Namen wurde eine Entfremdung der Menschen beiderseits der neu gezogenen Grenze angestrebt. Erst seit der Unabhängigkeit der Ukraine dürfen sich Rumänen dort wieder als solche bezeichnen.

Die Nationalitätsspalte im Pass schaffte die Ukraine zwar sofort nach der Unabhängigkeit ab, doch beklagt Petrasch, dass sich die Moldauer nicht als Rumänen bezeichnen und kein Interesse an einer effektiveren rumänischen Minderheitenpolitik zeigen. Das Kulturinstitut Eudoxiu Hurmuzachi in Tscherniwzi ist aber gleichermaßen für Rumänen und Moldauer offen.

Gerade junge Vertreter der rumänischen Minderheit wandern seit 1991 nach Rumänien aus. Dies hat sich 2007 durch den EU-Beitritt Rumäniens noch verstärkt. Die verbliebenen Rumänen haben meistens die doppelte Staatsbürgerschaft. Durch die Abwanderung vieler qualifizierter Rumänen aus der Ukraine fehlen Fachkräfte, vielen rumänischen Schulen in der Ukraine fehlen Lehrer. Seit 1991 nahm die Zahl der auf Rumänisch unterrichtenden Schulen stetig ab. Dies ist einerseits auf abwandernde Lehrer zurückzuführen, doch fühlen sich viele Rumänen auch durch die Forderungen national gesinnter ukrainischer Parteien eingeschüchtert, Ukrainisch müsse einzige Unterrichtssprache sein. Dieser Anspruch gehörte zur Agenda des ehemaligen ukrainischen Präsidenten Wiktor Juschtschenko, was Vertreter der rumänischen Minderheit damals in die Arme der Partei der Regionen (PdR) des ehemaligen Präsidenten Janukowytsch trieb, von der sie sich eine bessere Minderheitenpolitik versprachen. Zusammen mit den Stimmen vieler der in Tscherniwzi lebenden Russisch sprechenden Bürger zog ein für die PdR kandidierender Rumäne ins Kiewer Parlament, und Tscherniwzi wurde zur Hochburg der Partei im Südwesten der Ukraine. Zur von den meisten Rumänen gewünschten EU-Integration kam es unter Janukowytsch nicht, auch wenn seine Regierung den ukrainischen Rumänen die 10\%-Minderheitensprachregelung brachte, die Rumänisch zur Amtssprache in mehreren Gemeinden der Region Tscherniwzi machte.

Für die ukrainischen Rumänen bedeutet EU-Integration der Ukraine vor allem einen intensiven Austausch mit dem EU-Mitglied Rumänien und bessere Reisebedingungen. Eugen Petrasch setzt große Hoffnungen auf die nach dem Maidan gewählte Regierung und ihren proeuropäischen Kurs. Einige Ziele seien bereits im letzten Jahr erreicht worden, wie der Wegfall der Visumspflicht für Bewohner grenznaher Gebiete. Davon profitieren etwa die Bewohner des zu $80 \%$ von Rumänen bewohnten Kreises Hertsa in der Region Tscherniwzi, direkt an der rumänischen Grenze. Die Annäherung zwischen der Ukraine und Rumänien nach dem Maidan macht auch grenzüberschreitende wirtschaftliche und kulturelle Kooperation mög- 
lich. Der in Tiraspol (im mit russischer Unterstützung von Moldau abgespaltenen Transnistrien) aufgewachsene ukrainische Präsident Poroschenko hielt vor Vertretern der rumänischen Minderheit in Tscherniwzi eine Rede auf Rumänisch und eröffnete laut Petrasch ein ganz neues Kapitel in den Beziehungen zwischen der Ukraine und ihrer rumänischen Minderheit. Ex-Präsident Juschtschenko ist nach Aussage Petraschs noch durch diskriminierende Moldauerwitze aufgefallen. Trotz des aktuell entspannten Klimas zwischen der Ukraine und Rumänien bleiben Probleme wie das der rumänischen Schulen. Artur Kadelnyk ist bukowinischer Rumäne, doch sieht er seine Zukunft in der Ukraine. Während ältere Menschen der Zugehörigkeit der Nordbukowina zum rumänischen Staat nachtrauerten und die unrechtmäßige Annexion durch die Sowjetunion sowie die folgende Deportation Tausender Rumänen beklagen, sehen sich jüngere ukrainische Rumänen wie Kadelnyk als Ukrainer rumänisch-moldauischer Ethnizität. Das ukrainische Verständnis von Nation konstituiere sich heute jenseits des Begriffes der Ethnizität. Als Mitglied des "Klubs junger Reformatoren«, welcher Diskussionen zwischen Studenten und Politikern der Ukraine in Tscherniwzi organisiert, ist Kadelnyk in der ukrainischen Zivilgesellschaft aktiv. In seinem Heimatdorf in der Region Tscherniwzi gab es keine rumänische Schule. Dennoch nahm er außerhalb des regulären Unterrichts zusätzlich rumänische Schulstunden, die die Schule anbot. Kadelnyk sieht es als seine staatsbürgerliche Pflicht, Ukrainisch zu lernen. Dass man als Angehöriger der rumänischen Minderheit Anspruch auf zusätzlichen Unterricht auf Rumänisch hat, ist für Kadelnyk Synonym dafür, dass der Vorwurf der "Ukrainisierung" nicht haltbar sei. Ein Problem der "Ukrainisierung" besteht laut Kadelnyk vielmehr für manche Politiker in Rumänien, die sich zu Verteidigern der sich angeblich in Gefahr befindenden rumänischen Minderheit in der Ukraine stilisieren möchten. Diese Karte spielte etwa im rumänischen Wahlkampf 2014 der sozialdemokratische Präsidentschaftskandidat Victor Ponta, der gegenüber seinem deutschstämmigen Kontrahenten Klaus Johannis seine "wahre« rumänische Identität hervorheben wollte. Auch mehrere rechte Parteien in Rumänien, wie die bis 2008 im rumänischen Parlament eine große Rolle spielende "Großrumänien-Partei«, welche eine Rückgabe der Nordbukowina durch die Ukraine forderte, befeuern immer wieder die Diskussion um eine Diskriminierung der rumänischen Minderheit in der Ukraine.

Seit Beginn des Krieges in der Ostukraine werden auch ukrainische Rumänen einberufen, um gegen die von Russland dirigierten und aufgerüsteten sog. Separatisten zu kämpfen. Am 8. Februar 2015 sprach der russische Außenminister Sergej Lawrow bei der Münchener
Sicherheitskonferenz darüber, dass Vertreter der ungarischen und rumänischen Minderheiten in der Ukraine der sogenannten "positiven Diskriminierung« unterlägen, wenn es um die Mobilisierung in die ukrainische Armee gehe. In der von den russischen Staatsmedien betriebenen Propaganda wird die ukrainische Armee häufig als eine Truppe dargestellt, die von angeblich unter amerikanischem Einfluss stehenden Kommandeuren verheizt werde. Zwischen den Zeilen wirft Lawrow der ukrainischen Regierung, die in der russischen Rhetorik je nach Berichtssujet mal »illegitim«, mal »Partner« ist, vor, sich so der Minderheiten des Landes zu entledigen. Von solchen absurden Behauptungen wollen Eugen Petrasch oder Artur Kadelnyk nichts wissen. Selbst in Rumänien wird zu diesem Vorwurf keine Stimme laut. Stattdessen produzierte das einflussreiche rumänische Nachrichtenblatt »Adevarul« einen Dokumentarfilm über ethnische Rumänen in der ukrainischen Armee. Diese sprechen dort über ihren Alltag, ihre rumänische Identität, aber auch über die Notwendigkeit des Kampfes in der ukrainischen Armee. Von der Entwicklung einer stabilen und demokratischen Ukraine hänge schließlich auch die Zukunft der rumänischen Minderheit in diesem Land ab.

\section{Die Ungarn Transkarpatiens}

In der westukrainischen Region Transkarpatien leben laut der Volkszählung von 2001150.000 Ungarn - 12\% der Bevölkerung der Region. Mit 156.000 Personen ungarischer Herkunft in der gesamten Ukraine beträgt ihre Zahl 0,32\% der Gesamtbevölkerung. Auch in der Region Transkarpatien gibt es Kreise und einzelne Ortschaften, in denen Ungarisch zweite Amtssprache ist. Im Kreis Berehowe leben fast zur Hälfte Ungarn, viele Straßenschilder im Hauptort sind nur auf Ungarisch beschriftet. In Solotwino, einem südlichen Kreis der Region Transkarpatien, sind die Hinweisschilder sogar dreisprachig, da dort auch eine rumänische Minderheit lebt. Aufgrund des Zuzugs aus anderen Regionen der Sowjetunion sprechen auch viele Menschen in der Region Russisch, oft die interkulturelle Verkehrssprache. Obwohl in Transkarpatien deutlich weniger Ungarn leben als Rumänen in der Region Tscherniwzi, haben sich die Ungarn in einer Partei organisiert: der »Partei der Ungarn der Ukraine«. Ziel dieser Partei ist eine ungarische Autonomie in der Region Transkarpatien, trotz des nicht ausreichenden Anteils der Ungarn in der gesamten Region. Unterstützt werden sie in dieser Forderung von den rechten Parteien Ungarns, Fidesz und Jobbik. Der russische Außenminister Sergej Lawrow kritisierte die ukrainische Regierung dafür, den Ungarn Transkarpatiens keine Autonomie zu gewähren, und forderte eine Verfassungsänderung, nach der die ungarische Minderheit einen Abgeordneten ins ukrainische Parlament 
schicken dürfte. Allerdings wurde die russische Diplomatie erst nach dem Maidan und dem damit verbundenen Regierungswechsel in der Ukraine zur selbsternannten Verteidigerin der Minderheiten in der Ukraine.

Die alltäglichen Probleme der Ungarn Transkarpatiens ähneln denen der Rumänen in der Region Tscherniwzi. Seit 1991 wandern viele Ungarn nach Ungarn oder in andere EU-Staaten aus. Seit der Unabhängigkeit der Ukraine haben die Regierungen Ungarns ihre Staatsbürgerschaft großzügig an ethnische Ungarn in der Ukraine vergeben. Daher liegt es auch im Interesse ungarischer Politiker, im Wahlkampf über Sorgen und Probleme der ungarischen Minderheit in der Ukraine zu reden. Bei der Europawahl 2014 trat eine Vertreterin der ungarischen Minderheit der Ukraine, Andrea Bocskor, für die ungarische Fidesz-Partei an und wurde ins Europaparlament gewählt. Dies macht sie zum ersten Mitglied des Europaparlaments mit (zusätzlich) ukrainischer Staatsbürgerschaft. Die Motivation für die Abwanderung ist vor allem wirtschaftlicher Natur. Von einer offenen Diskriminierung der Ungarn in der Ukraine kann keine Rede sein, genauso wenig wie die ungarische Minderheit der Ukraine geschlossen den Anspruch der rechtsorientierten "Partei der Ungarn in der Ukraine« anerkennt, ihre Interessen zu vertreten. Auch das stellt den Anspruch dieser Partei in Frage, eine Verfassungsänderung zu fordern, die ihnen einen Platz in der Obersten Rada gewähren würde. Zudem ziehen viele junge Ungarn statt nach Ungarn nach Kiew und sehen ihre Zukunft als Bürger der Ukraine.

Olexandr Solontaj ist Experte des »Instituts politischer Aufklärung« in Kiew. Er stammt aus Transkarpatien. Sein Vater ist ethnischer Ungar. Auch wenn er sich als ukrainischer Bürger sieht und sich für eine proeuropäische demokratische Ukraine politisch engagiert, spricht er in einem Interview mit der ukrainischen Online-Zeitung "Glavcom« offen über die Ängste der ungarischen Minderheit in der Ukraine. Auch wenn der Zwischenfall von Mukatschewe am 12. Juli 2015 einen ganz anderen Hintergrund hatte, habe er unter den ukrainischen Ungarn zu Ängsten vor einer Destabilisierung der Region geführt. Die Aussagen des ungarischen Geheimdienstchefs, ungarische Agenten würden die Interessen Budapests und der ungarischen Minderheit in der Ukraine verteidigen, führten zu diplomatischen Verstimmungen. Doch auch Solontaj räumt ein, dass die ungarische Minderheit in der Ukraine sich stärker an Budapest orientieren und um ungarisches Eingreifen bitten könnte, sollte es der ukrainischen Regierung nicht gelingen, Sicherheit in der Region zu garantieren.
Die Beziehungen zwischen der Ukraine und Ungarn sind kühl, der ungarische Ministerpräsident Viktor Orban lud den russischen Präsidenten Wladimir Putin jedoch trotz EU-Sanktionen und Ukrainekrise zum Staatsbesuch nach Budapest ein. "Patriotisch" gesinnte russische Spitzenpolitiker sehen wegen deren konservativer Werte in der Fidesz-Partei Seelenverwandte. Der russische Politiker Wladimir Zhirinowskij, der für seine rechtspopulistischen Aussagen bekannt ist, wetterte gar, dass man die Ukraine aufteilen und Transkarpatien an Ungarn abtreten sollte. Dabei gehörte Transkarpatien während der Habsburger Monarchie zwar zum Königreich Ungarn, in der Zwischenkriegszeit jedoch zur Tschechoslowakei. Prag gewährte der mehrheitlich von Ukrainern bewohnten Region eine breite Autonomie. Die Region bekam den Namen »Karpaten-Rus». 1939 wurde Transkarpatien von der dem Dritten Reich nahestehenden Horthy-Regierung Ungarns annektiert, nach 1945 jedoch nicht von der Sowjetunion an die wiederhergestellte Tschechoslowakei zurückgegeben, sondern der UdSSR einverleibt. Eine Bezeichnung von Transkarpatien als historisch zu Ungarn gehörendes Gebiet ist also schon historisch schief. Trotz der persönlichen Annäherung ungarischer und russischer Politiker trägt Ungarn die europäischen Sanktionen gegen Russland mit und ist darüber hinaus am sogenannten »ReverseFlow» beteiligt, der Gas aus Ungarn in die Ukraine leitet.

\section{Fazit}

Die ethnischen Minderheiten der Ukraine spielen in den außenpolitischen Beziehungen der Ukraine zu ihren Nachbarn häufig eine Rolle. Dabei hängen diese zwischenstaatlichen Beziehungen nicht zwangsweise von der tatsächlichen Lage der Minderheiten in der Ukraine ab. Die ungarische Fidesz-Partei oder der rumänische Präsidentschaftswahlkämpfer Victor Ponta sind Beispiele dafür, wie mitteleuropäische Politiker versuchen, ihre Zustimmungswerte dadurch zu steigern, dass sie sich als Verteidiger ihrer jeweiligen Minderheit in der Ukraine stilisieren. Die ukrainische Regierung erklärt, dass die angekündigte Dezentralisierungsreform den jeweiligen Minderheiten in ihren Siedlungsgebieten mehr Gestaltungsrechte garantieren werde. Seit der Unabhängigkeit gab es auch Überlegungen, ein Zweikammerparlament einzuführen, um den Regionen mehr Mitspracherechte in der gesamtukrainischen Politik zu geben. Eine solch tiefgreifende Reform steht gegenwärtig jedoch nicht auf der Reformagenda.

Über den Autor:

Oleg Friesen ist Masterstudent der Osteuropawissenschaften an der LMU München und war im Juni/Juli 2014 sowie von März bis Juni 2015 Praktikant im Auslandsbüro der Friedrich-Naumann-Stiftung in Kiew. 


\section{2. - 25. Oktober 2015}

\begin{tabular}{|c|c|}
\hline 12.10 .2015 & $\begin{array}{l}\text { Pro-ukrainische Aktivisten erklären, dass eine von vier Stromtrassen, die Elektrizität vom ukrainischen Festland } \\
\text { auf die Krim transportieren, ihren Betrieb eingestellt habe. Medien berichten, die Leitung sei von Unbekannten } \\
\text { beschädigt worden und könne zurzeit aus Sicherheitsgründen nicht repariert werden, da sich etwa einhundert } \\
\text { Aktivisten am Ort befänden. Energieminister Wolodymyr Demtschyschyn erklärt unterdessen, dass über alle vier } \\
\text { Trassen Strom geliefert werden solle. Einer Blockade aus politischen Gründen erteilt er eine Absage. }\end{array}$ \\
\hline 12.10 .2015 & $\begin{array}{l}\text { n Mitarbeiter des krimtatarischen Fernsehsenders ART wird auf der Krim zu zweieinhalb Jahren Gefängnis } \\
\text { ff Bewährung verurteilt. Ihm war die Teilnahme an »Massenunruhen« Ende Februar } 2014 \text { vorgeworfen worden. }\end{array}$ \\
\hline 12.10 .2015 & $\begin{array}{l}\text { Die Separatisten der »Volksrepublik Luhansk« verschieben die zuvor für den 1. November } 2015 \text { angesetzten Lokal- } \\
\text { wahlen auf den 21. Februar } 2016 .\end{array}$ \\
\hline 12.10 .2015 & $\begin{array}{l}\text { Die Ukraine sperrt ab dem 25. Oktober } 2015 \text { ihren Luftraum vollständig für russische Fluggesellschaften. Dies } \\
\text { sei eine Reaktion auf das Verbot für fünf ukrainische Fluggesellschaften, russische Flughäfen anzusteuern. Russ- } \\
\text { land hatte das Verbot wiederum auf eine gleichlautende Sanktion der Ukraine hin erlassen. }\end{array}$ \\
\hline 13.10 .2015 & $\begin{array}{l}\text { Ein krimtatarischer Koordinator der Kampagne zur Energieblockade der Krim erklärt, man strebe die komplette } \\
\text { Abtrennung der von Russland annektierten Halbinsel vom ukrainischen Stromnetz an. Dazu erwarte man vom } \\
\text { Parlament ein entsprechendes Gesetz. }\end{array}$ \\
\hline 13.10 .2015 & $\begin{array}{l}\text { Die Generalstaatsanwaltschaft erklärt, sie untersuche die Ereignisse des 20. Februar 2014. Nach Informationen } \\
\text { verschiedener Medien (darunter die britische BBC und die ARD) wurden während der Maidan-Proteste aus dem } \\
\text { damals von der Opposition besetzten Hotel Ukraina Schüsse auf Demonstranten abgegeben. Mehrere Aktivis- } \\
\text { ten der rechtsradikalen Partei Freiheit werden dazu als Zeugen befragt. }\end{array}$ \\
\hline 13.10 .2015 & $\begin{array}{l}\text { Einer Umfrage des Kiewer Internationalen Instituts für Soziologie und des Internationalen Fonds für Wahlsys- } \\
\text { teme zufolge haben } 40 \% \text { der Ukrainer in den vergangenen zwölf Monaten ein Bestechungsgeld gezahlt. } 32 \% \\
\text { zahlten für die Aufnahme im Krankenhaus, neun Prozent für die Bearbeitung von Dokumenten, und sechs Pro- } \\
\text { zent leisteten eine Bestechungszahlung, um einer Geldbuße im Straßenverkehr zu entgehen. }\end{array}$ \\
\hline 13.10 .2015 & $\begin{array}{l}\text { Das niederländische Ermittlerteam, das den Absturz des Passagierflugzeugs der Malaysia Airlines am 17. Juli } 2014 \\
\text { untersucht hat, veröffentlicht seinen Abschlussbericht. Darin wird zweifelsfrei bestätigt, dass das Flugzeug von einer } \\
\text { Boden-Luft-Rakete russischer Bauart des Typs BUK getroffen wurde. Der Bericht macht keine genauen Anga- } \\
\text { ben über den Ort des Abschusses, wenngleich er feststellt, dass der größte Teil des infrage kommenden Gebietes } \\
\text { unter der Kontrolle der Separatisten war. Der Bericht betont außerdem, dass es für den ukrainischen Staat aus- } \\
\text { reichend Gründe gegeben habe, den Luftraum auch in dieser Höhe zu schließen. }\end{array}$ \\
\hline 14.10 .2015 & $\begin{array}{l}\text { Der Generalstab der ukrainischen Armee erklärt, ein Soldat sei durch Beschuss ums Leben gekommen. Am Abend } \\
\text { teilt der Stab mit, ansonsten werde der Waffenstillstand eingehalten, die Lage sei ruhig. }\end{array}$ \\
\hline 14.10 .2015 & $\begin{array}{l}\text { Präsident Petro Poroschenko erklärt, dass der ukrainische Staat über zehn Milliarden Hrywnja (etwa } 400 \text { Mil- } \\
\text { lionen Euro) für den Wiederaufbau der zerstörten Infrastruktur im Donbass zurückgestellt habe. Dieser Betrag } \\
\text { werde um das Vier- bis Fünffache von europäischen Staaten und den USA erweitert. Bundeskanzlerin Angela } \\
\text { Merkel habe } 500 \text { Millionen Euro zugesagt. }\end{array}$ \\
\hline 15.10 .2015 & $\begin{array}{l}\text { Präsident Petro Poroschenko verleiht drei Soldaten der ukrainischen Armee den Orden »Held der Ukraine«, einem } \\
\text { davon posthum. Der verstorbene Ihor Sinytsch habe während der Kämpfe um den Donezker Flughafen } 50 \text { bis } 70 \\
\text { Menschen das Leben gerettet. »Held der Ukraine« ist die höchste Auszeichnung des Staates. }\end{array}$ \\
\hline 15.10 .2015 & $\begin{array}{l}\text { Während der ukrainische Generalstab meldet, dass beide Seiten die schweren Waffen abziehen, berichtet die } \\
\text { OSZE-Beobachtermission, dass sowohl die ukrainische Armee als auch die Separatisten einzelne Waffen entge- } \\
\text { gen den Vereinbarungen nicht abgezogen hätten. Vertreter der Mission beklagen außerdem, dass Bewaffnete ihr } \\
\text { in der Region Luhansk den Zutritt zu Grenzübergängen verwehrt hätten. }\end{array}$ \\
\hline 15.10 .2015 & $\begin{array}{l}\text { Die OSZE teilt mit, dass bereits einige Tausend Menschen, die vor der Gewalt im Osten der Ukraine geflohen } \\
\text { waren, in ihre Häuser im Donbass zurückgekehrt seien. }\end{array}$ \\
\hline 15.10 .2015 & $\begin{array}{l}177 \text { von } 192 \text { Mitgliedsstaaten der Vereinten Nationen wählen die Ukraine zum nichtständigen Mitglied im UN- } \\
\text { Sicherheitsrat für die Jahre } 2016 \text { und } 2017 .\end{array}$ \\
\hline 15.10 .2015 & $\begin{array}{l}\text { Der Journalist Michail Tkatsch berichtet von einem Versuch der Kiewer Abteilung des Inlandsgeheimdienstes } \\
\text { SBU, die Ausstrahlung einer Reportage zu verhindern. Der Film zeige die teuren Privatfahrzeuge einiger Mit- } \\
\text { arbeiter des Dienstes. Bereits während der Dreharbeiten hätten Mitarbeiter des SBU die Dreharbeiten bedroht. }\end{array}$ \\
\hline
\end{tabular}




\begin{tabular}{|c|c|}
\hline 16.10.2015 & $\begin{array}{l}\text { in Vertreter des Verteidigungsministeriums erklärt, ukrainische Bürger hätten der ukrainischen Armee im Laufe } \\
\text { es Jahres bereits etwa 6,5 Millionen Hrywnja (etwa 260.000 Euro) gespendet. Das Verteidigungsministerium } \\
\text { irbt in einer Kampagne um Spenden für die Unterstützung der »Anti-Terror-Operation«. }\end{array}$ \\
\hline 16.10.2015 & $\begin{array}{l}\text { In einer Umfrage der } » \text { Rating } «-\text { Gruppe geben } 68 \% \text { der Befragten an, die Reformen in der Ukraine bewegten } \\
\text { sich in die falsche Richtung. Im Juli waren es } 72 \% \text {, im April } 2014 \text { allerdings noch } 48 \% \text {. Elf Prozent geben an, } \\
\text { die Tätigkeit des Parlaments zu unterstützen, } 81 \% \text { sehen sie kritisch. Die Arbeit des Präsidenten begrüßen } 24 \% \\
\text { der Befragten, } 69 \% \text { lehnen sie ab. }\end{array}$ \\
\hline 16.10 .2015 & $\begin{array}{l}\text { Einer Umfrage der »Rating«-Gruppe zufolge sind } 48 \% \text { der Ukrainer für einen Beitritt des Landes zur Nato, } 28 \% \\
\text { sprechen sich dagegen aus. Die Zustimmung zu einem Beitritt befindet sich damit auf einem Rekordhoch. Im } \\
\text { März } 2014 \text { hatte sie noch bei } 34 \% \text { gelegen. }\end{array}$ \\
\hline 17.10 .2015 & $\begin{array}{l}\text { Die Separatisten der "Volksrepublik Donezk« kündigen an, am 21. Oktober } 2015 \text { mit dem Abzug der schweren } \\
\text { Waffen von Kalibern unter } 100 \text { Millimetern zu beginnen. Zunächst war für den Beginn der 18. Oktober } 2015 \\
\text { genannt worden. Sowohl die ukrainische Armee als auch die Separatisten der »Volksrepublik Luhansk» haben } \\
\text { bereits mit dem Abzug begonnen. }\end{array}$ \\
\hline 17.10 .2015 & $\begin{array}{l}\text { Vertreter der ukrainischen Armee erklären, eine ihrer Positionen sei mit Kleinwaffen angegriffen worden. Zuvor } \\
\text { hatte es für mehrere Tage keine Gefechte gegeben. }\end{array}$ \\
\hline 17.10 .2015 & Bei Odessa verunglückt ein Ausflugsschiff. 14 Menschen kommen ums Leben. \\
\hline 18.10.2015 & $\begin{array}{l}\text { äsident Petro Poroschenko spricht sich dafür aus, die Strafen für Stimmenkauf bei Wahlen zu verschärfen. So } \\
\text { llten Kandidaten, die sich des gerichtlich erwiesenen Stimmenkaufs schuldig gemacht hätten, von der Wahl } \\
\text { sgeschlossen werden. Er ruft die Regierung dazu auf, entsprechende Gesetze zu initiieren. }\end{array}$ \\
\hline 19.10.2015 & $\begin{array}{l}\text { in Vertreter der Organisation Kraft der Menschen beklagt, dass es in Mariupol bei der Vorbereitung der Lokal- } \\
\text { ahlen Unregelmäßigkeiten gegeben habe. Die Wahlzettel seien in einer Druckerei produziert worden, die dem } \\
\text { nflussreichen Unternehmer Rinat Achmetow gehöre. Laut Angaben der Organisation waren während des Drucks } \\
\text { 1sschließlich Mitarbeiter der Druckerei anwesend, es habe keine Kontrolle stattgefunden. Der Vorsitzende der } \\
\text { onezker Gebietsverwaltung Pawlo Schebriwskyj erklärt später, er erwäge, die Zentrale Wahlkommission um } \\
\text { ne Absage der für den 25. Oktober } 2015 \text { geplanten Lokalwahlen in Mariupol zu ersuchen. Immer wieder gebe } \\
\text { Versuche, die Arbeit der städtischen Wahlkommission zu blockieren. }\end{array}$ \\
\hline 19.10.2015 & $\begin{array}{l}\text { Im Gebiet um Kiew kommt es vermehrt zu Torfbränden. Zurzeit brennen etwa } 122 \text { Hektar Land. Hunderte Feu- } \\
\text { erwehrleute sind im Einsatz. }\end{array}$ \\
\hline 19.10.2015 & $\begin{array}{l}\text { ne britische Anwaltskanzlei erklärt, der ehemalige Präsident Wiktor Janukowytsch habe Klage beim Europäi- } \\
\text { hen Gerichtshof für Menschenrechte eingereicht. Die Ukraine habe u. a. sein Recht auf einen fairen Gerichts- } \\
\text { ozess verletzt und ihn aufgrund seines politischen Status und seiner politischen Ansichten diskriminiert. }\end{array}$ \\
\hline 19.10.2015 & $\begin{array}{l}\text { Infrastrukturminister Andryj Piwowarskyj erklärt, die ukrainische Flugaufsichtsbehörde sei auf Anfrage der rus- } \\
\text { sischen Seite zu Verhandlungen über den Luftverkehr zwischen beiden Ländern bereit. Am 25. September } 2015 \\
\text { hatte die Ukraine im Zuge ihrer Sanktionen gegen Russland einigen russischen Fluggesellschaften verboten, } \\
\text { ukrainische Ziele anzufliegen. Russland hatte daraufhin mit einem entsprechenden Verbot reagiert. Beide Ver- } \\
\text { bote waren in der Folge auf den gesamten Flugverkehr ausgedehnt worden. }\end{array}$ \\
\hline 20.10 .2015 & $\begin{array}{l}\text { Ukrainische Quellen berichten, dass mehrere Separatisten bei Explosionen während Aufräumarbeiten in Minen- } \\
\text { feldern ums Leben gekommen seien. Auch ein Soldat der ukrainischen Armee wird durch eine Mine getötet. }\end{array}$ \\
\hline 20.10.2015 & $\begin{array}{l}\text { Die Generalstaatsanwaltschaft lädt den ehemaligen Chef des Inlandsgeheimdienstes SBU, Walentyn Nalywajt- } \\
\text { schenko, vor. Er hatte wiederholt öffentlich behauptet, dass Angehörige des russischen Inlandsgeheimdienstes FSB } \\
\text { mit den Schüssen auf Demonstranten während der Demonstrationen auf dem Maidan im Februar } 2014 \text { in Ver- } \\
\text { bindung ständen. Generalstaatsanwalt Wiktor Schokin erklärt, er habe keine Informationen, um diese Behaup- } \\
\text { tung zu stützen. Wenn Nalywajtschenko Belege dafür habe, solle er sie der Staatsanwaltschaft aushändigen. }\end{array}$ \\
\hline 21.10.2015 & $\begin{array}{l}\text { Ministerpräsident Arsenij Jazenjuk bittet das Parlament, einige Minister und zivilgesellschaftliche Organisationen } \\
\text { öffentlich darum, ihm Vorschläge für die Ausgestaltung eines neu zu schaffenden Ministeramtes für die »Anti-Ter- } \\
\text { ror-Operation« zu unterbreiten. Die Verfassung erlaube es, solche Posten ohne eigenes Ministerium einzurichten. }\end{array}$ \\
\hline 21.10 .2015 & $\begin{array}{l}\text { Ministerpräsident Arsenij Jazenjuk erklärt, die Lustration genannte Überprüfung hoher Beamter des staatlichen } \\
\text { Finanzdienstes auf Korruptionsdelikte habe ergeben, dass } 42 \% \text { der Beamten der Führungsebene sofort entlas- } \\
\text { sen werden müssten. }\end{array}$ \\
\hline 21.10 .2015 & $\begin{array}{l}\text { Präsident Petro Poroschenko erklärt, die Separatisten der „Volksrepublik Donezk« hätten mit dem Abzug der } \\
\text { schweren Waffen von Kalibern unter } 100 \text { Millimetern begonnen. Dies hatte der »Ministerpräsident« der »Volks- } \\
\text { republik«, Oleksandr Sachartschenko, zuvor angekündigt. }\end{array}$ \\
\hline
\end{tabular}




\begin{tabular}{|c|c|}
\hline 21.10.2015 & $\begin{array}{l}\text { Ein Vertreter des Internationalen Währungsfonds (IWF) erklärt, dass ein Gesetz zur Reformierung des Steuer- } \\
\text { systems, das zurzeit im Parlament liegt, das Kreditprogramm des IWF für die Ukraine gefährden könne. Die im } \\
\text { Gesetz vorgesehene Steuersenkung sei nicht ausreichend durch Einsparungen gedeckt. Die Abstimmung über das } \\
\text { Gesetz wird infolge der Kritik des IWF zunächst auf den 26. Oktober } 2015 \text { verschoben. }\end{array}$ \\
\hline 22.10.2015 & $\begin{array}{l}\text { Die OSZE-Beobachtermission berichtet, dass in der Region um das von Separatisten kontrollierte Donezk wei- } \\
\text { terhin vereinzelt Gewehrsalven und Explosionen zu hören seien. }\end{array}$ \\
\hline 22.10.2015 & Vertreter der "Volksrepublik Donezk« erklären, alle Panzer seien von der Frontlinie abgezogen worden. \\
\hline 22.10.2015 & $\begin{array}{l}\text { Die Zentralbank hebt die Beschränkungen für Kapitalverkehr in Fremdwährungen teilweise auf. Sie gestat- } \\
\text { tet ukrainischen Banken wieder, Fremdwährung von natürlichen Personen in unbegrenzter Höhe einzukaufen. }\end{array}$ \\
\hline 23.10.2015 & $\begin{array}{l}\text { Die Venedig-Kommission des Europarates bewilligt einen Gesetzentwurf zur Reform des Justizsystems. Das } \\
\text { Gesetz war von zivilgesellschaftlichen Organisationen entwickelt worden und sieht unter anderem die Neube- } \\
\text { stellung von Richtern vor. Die Kommission erklärt jedoch, dass nicht alle Richter ausgetauscht werden dürften. } \\
\text { Vielmehr seien sie auf ihre Eignung zu prüfen, was in manchen Fällen auch zum Austausch führen könne. Auch } \\
\text { Präsident Petro Poroschenko äußert Zustimmung zu dem Gesetz. }\end{array}$ \\
\hline 24.10 .2015 & $\begin{array}{l}\text { Vertreter der Organisation Ärzte ohne Grenzen berichten, dass die »Volksrepublik Donezk» der Organisation die } \\
\text { Akkreditierung für die Arbeit auf dem von den Separatisten kontrollierten Territorium entzogen habe. Man sei } \\
\text { ohne Angabe von Gründen aufgefordert worden, das Gebiet schnellstmöglich zu verlassen. }\end{array}$ \\
\hline 24.10.2015 & $\begin{array}{l}\text { Nach Aussagen des Vorsitzenden der Donezker Gebietsverwaltung, Pawlo Schebriwskyj, ist die Lage unmittel- } \\
\text { bar vor den Lokalwahlen in zwei Städten des Donbass angespannt. In Krasnoarmijsk und in Mariupol habe es } \\
\text { Unregelmäßigkeiten beim Druck der Wahlzettel gegeben. In Mariupol erklärt unterdessen die lokale Wahlkom- } \\
\text { mission, dass man die Wahlzettel aus der Druckerei nicht abholen werde, da sie Fehler in der Bezeichnung meh- } \\
\text { rerer Parteien enthielten. Bis zur Wahl am nächsten Tag könnten keine korrekten Stimmzettel mehr gedruckt } \\
\text { werden, die Durchführung der Wahl sei daher akut gefährdet. Die Kommission bittet das Zentrale Wahlkomi- } \\
\text { tee, den Wahltermin zu verschieben. }\end{array}$ \\
\hline 25.10.2015 & $\begin{array}{l}\text { Auf dem gesamten Gebiet der Ukraine mit Ausnahme der von Separatisten kontrollierten Territorien im Donbass } \\
\text { finden Lokalwahlen statt. Laut der Wahlbeobachterorganisation OPORA beträgt die durchschnittliche Wahlbe- } \\
\text { teiligung } 47 \% \text {. Im Osten liegt sie mit } 43 \% \text { etwas darunter. Aufgrund fehlender Stimmzettel können die Lokal- } \\
\text { wahlen in Mariupol nicht durchgeführt werden. Trotz gegenteiliger Mitteilungen im Tagesverlauf finden auch } \\
\text { in Kransoarmijsk aufgrund von Problemen bei der Verteilung der Stimmzettel keine Wahlen statt. Präsident } \\
\text { Petro Poroschenko ruft das Parlament dazu auf, noch im Jahr } 2015 \text { eine Gesetzesgrundlage für die nachträgliche } \\
\text { Durchführung der Wahlen zu schaffen. }\end{array}$ \\
\hline
\end{tabular}

Die Chronik wird zeitnah erstellt und basiert ausschließlich auf im Internet frei zugänglichen Quellen. Die Redaktion bemüht sich, bei jeder Meldung die ursprüngliche Quelle eindeutig zu nennen. Aufgrund der großen Zahl von manipulierten und falschen Meldungen kann die Redaktion der Ukraine-Analysen keine Gewähr für die Richtigkeit der Angaben übernehmen.

Zusammengestellt von Jan Matti Dollbaum

Sie können die gesamte Chronik seit Februar 2006 auch auf <http://www.laender-analysen.de/ukraine/> unter dem Link »Chronik«lesen.

Herausgeber: Forschungsstelle Osteuropa an der Universität Bremen und Deutsche Gesellschaft für Osteuropakunde

Die Meinungen, die in den Ukraine-Analysen geäußert werden, geben ausschließlich die Auffassung der Autoren wieder.

Abdruck und sonstige publizistische Nutzung sind nach Rücksprache mit der Redaktion gestattet. Verantwortlicher Redakteur für diese Ausgabe: Katerina Bosko (geb. Malygina) Satz: Matthias Neumann

Ukraine-Analysen-Layout: Cengiz Kibaroglu, Matthias Neumann und Michael Clemens

Alle Ausgaben der Ukraine-Analysen sind mit Themen- und Autorenindex archiviert unter www.laender-analysen.de

Die Ukraine-Analysen werden im Rahmen eines Lizenzvertrages in das Internetangebot der Bundeszentrale für politische Bildung (www.bpb.de) aufgenommen

Die Ukraine-Analysen werden im Rahmen der Datenbank World Affairs Online (WAO) ausgewertet und sind im Portal IREON www.ireon-portal.de recherchierbar. ISSN 1862-555X @ 2015 by Forschungsstelle Osteuropa, Bremen

Forschungsstelle Osteuropa • Publikationsreferat • Klagenfurter Str. 3 - 28359 Bremen • Telefon: +49 421-218-69600 • Telefax: +49 421-218-69607 e-mail: publikationsreferat@osteuropa.uni-bremen.de•Internet-Adresse: http://www.laender-analysen.de/ukraine/ 


\section{Kostenlose E-Mail-Dienste der Forschungsstelle Osteuropa und ihrer Partner auf www.laender-analysen.de}

Die Länder-Analysen bieten regelmäßig im kostenlosen Abonnement kompetente Einschätzungen aktueller politischer, wirtschaftlicher, sozialer und kultureller Entwicklungen in Ostmitteleuropa und der GUS. Sie machen das Wissen, über das die wissenschaftliche Forschung in reichem Maße verfügt, für Politik, Wirtschaft, Medien und die interessierte Öffentlichkeit verfügbar. Autoren sind internationale Fachwissenschaftler und Experten.

Die einzelnen Länder-Analysen werden von der Forschungsstelle Osteuropa an der Universität Bremen und der Deutschen Gesellschaft für Osteuropakunde jeweils mit unterschiedlichen Partnern und Sponsoren herausgegeben. Die Redaktionen der Länder-Analysen bestehen aus Wissenschaftlern mit langjähriger Forschungserfahrung.

Die Länder-Analysen bieten regelmäßig Kurzanalysen zu aktuellen Themen, ergänzt um Grafiken und Tabellen sowie Dokumentationen. Zusätzlich gibt es eine Chronik aktueller Ereignisse. Alle Länder-Analysen sind auch mit Archiv und Indizes online verfügbar unter $<$ www.laender-analysen.de $>$.

\section{Belarus-Analysen}

Erscheinungsweise: zweimonatlich

Abonnement unter: <http://www.laender-analysen.de/belarus/>

\section{Caucasus Analytical Digest}

In englischer Sprache. Erscheinungsweise: monatlich

Abonnement unter: <http://www.css.ethz.ch/publications/newsletter_CAD_EN>

\section{Polen-Analysen}

Erscheinungsweise: zweimal monatlich

Abonnement unter: <http://www.deutsches-polen-institut.de/newsletter/polen-analysen/>

\section{Russland-Analysen}

Erscheinungsweise: zweiwöchentlich

Abonnement unter: <http://www.laender-analysen.de/russland/>

\section{Russian Analytical Digest}

In englischer Sprache. Erscheinungsweise: zweimal monatlich

Abonnement unter: <http://www.css.ethz.ch/publications/newsletter_RAD_EN>

\section{Ukraine-Analysen}

Erscheinungsweise: zweimal monatlich

Abonnement unter: <http://www.laender-analysen.de/ukraine/>

\section{Zentralasien-Analysen}

Erscheinungsweise: monatlich

Abonnement unter: <http://www.laender-analysen.de/zentralasien/>

\section{Bibliographische Dienste}

Die Bibliographien informieren über englisch- und deutschsprachige Neuerscheinungen zu Belarus, Russland, Ukraine sowie zu den zentralasiatischen und kaukasischen Staaten. Erfasst werden jeweils die Themenbereiche Politik, Außenpolitik, Wirtschaft und Soziales.

Erscheinungsweise: viermal jährlich

Abonnement unter: <http://www.laender-analysen.de/bibliographies/belarus.php>, <http://www.laender-analysen. de/bibliographies/russia.php>, <http://www.laender-analysen.de/bibliographies/ukraine.php>, <http://www.laenderanalysen.de/bibliographies/caucasus_ca.php> 